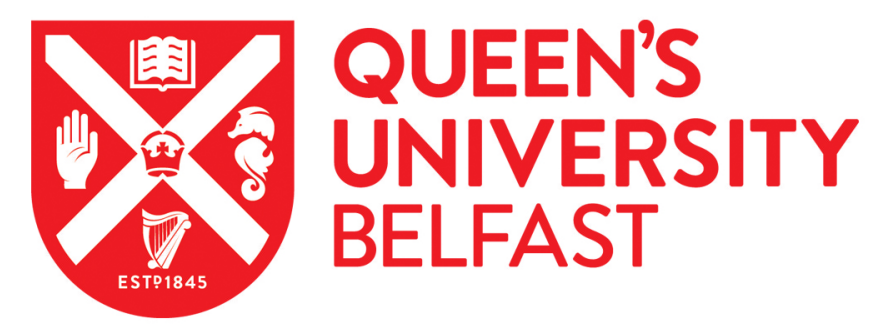

\title{
Axisymmetric structural optimization design and void control for selective laser melting
}

Stojanov, D., Wu, X., Falzon, B., \& Yan, W. (2017). Axisymmetric structural optimization design and void control for selective laser melting. Structural and Multidisciplinary Optimization, 1-17. https://doi.org/10.1007/s00158017-1700-x

\section{Published in:}

Structural and Multidisciplinary Optimization

\section{Document Version:}

Peer reviewed version

Queen's University Belfast - Research Portal:

Link to publication record in Queen's University Belfast Research Portal

\section{Publisher rights}

(C) 2017 Springer International Publishing AG. This work is made available online in accordance with the publisher's policies. Please refer to any applicable terms of use of the publisher.

\section{General rights}

Copyright for the publications made accessible via the Queen's University Belfast Research Portal is retained by the author(s) and / or other copyright owners and it is a condition of accessing these publications that users recognise and abide by the legal requirements associated with these rights.

Take down policy

The Research Portal is Queen's institutional repository that provides access to Queen's research output. Every effort has been made to ensure that content in the Research Portal does not infringe any person's rights, or applicable UK laws. If you discover content in the Research Portal that you believe breaches copyright or violates any law, please contact openaccess@qub.ac.uk. 


\title{
Axisymmetric structural optimization design and void control for selective laser melting
}

\author{
Daniel Stojanov, Xinhua Wu, Brian G. Falzon, Wenyi Yan
}

\begin{abstract}
Additive manufacturing processes, of which Selective Laser Melting (SLM) is one, provide an increased design freedom and the ability to build structures directly from CAD models. There is a growing interest in using optimization methods to design structures in place of manual designs. Three design optimization problems were addressed in this paper. The first related to axisymmetric structures and the other two addressing important design constraints when manufacturing using SLM. These solutions were developed and applied to a case study of a turbine containment ring. Firstly, many structural components such as a turbine containment ring are axisymmetric while they are subjected to a non-axisymmetric load. A solution was presented in this paper to generate optimized axisymmetric designs for a problem in which the mechanical model was not axisymmetric. The solution also worked equally well for generating a prismatic geometry with a uniform cross section, requiring no change in the procedure from axisymmetric designs to achieve this. Secondly, the SLM process experiences difficulties manufacturing structures with internal voids larger than a certain upper limit. A method was developed that allowed the designer to provide a value for this upper limit to the optimization method which would prevent the generation of internal voids larger than this value in any optimized design. The method calculated the sizes of all the voids and did not increase their size once they reached this limit. It was also aware of voids near each other, providing a minimum distance between them. Finally, in order to remove the metal powder, that fills the internal voids of structures built using SLM to reduce unnecessary weight, a method was developed to build paths to join the internal voids created during the optimization process. It allowed the analyst to nominate suitable path entrance locations from which powder could be removed, then found the shortest path connecting all voids and these locations. For axisymmetric structures it also distributed this path around the circumference to avoid generating weak points.
\end{abstract}

\section{Introduction}

Additive manufacturing technology is becoming increasingly popular as a manufacturing process (Hopkinson et al. 2006). This paper describes methods developed for the optimization of energy absorbing structures to be built using additive manufacturing (AM) technologies. There is a particular focus in this research on the Selective Laser Melting (SLM) technique. SLM progressively builds up a component by depositing layers of metal powder over a build plate. It uses a laser to melt and solidify regions within each layer, progressively building a structure defined in a computer aided design file provided to the computer controlling the process. This manufacturing process has the advantage that 
it allows for thin-walled regions, complex geometry, internal or not easily accessible features that would otherwise require access by tooling, and fast turnaround times for short production runs.

There is an inevitable change in the methods being used to design structures for SLM and other AM techniques, from those used for manufacturing techniques that existed previously. In many cases there is little or no additional cost to adding many types of complexity to a structure. There is interest in both building existing designs using additive manufacturing and designing new types of structures that will only be manufactured by this process. This has included building truss and scaffold structures using AM techniques (Brooks et al. 2005). Structures unique to AM include trusses made of a single piece of material with no joints between elements, that are each given a unique taper, and which can be mapped to the boundaries of a component to provide a stronger surface (Wang et al. 2005). This has been extended to a design methodology for designing truss structures for additive manufacturing, including an XML file format for the efficient exchange of these large degree of freedom truss structures (Chen 2006 and Rosen 2007). This has also been used in controlling the morphing of a wing to favourably alter its cross-section during the duration of an aircraft's mission (Chu et al. 2008). There are also applications of this technology to the medical industry. The literature features work to design truss structures to provide bone scaffold and to conform to the shape of bones from medical scans (Naing et al. 2005, Sun et al. 2005 and Challis et al. 2010). This has also included creating efficient representations of stochastic cellular material that closely mimics the characteristic of bone material (Schroeder et al. 2005).

Topology optimization methods have been shown to be effective and automated methods to optimize mechanical structures (Bendsøe 1989, Rozvany et al. 1992, Huang et al. 2006, Yoon and Kim 2007, Wu and Tseng 2010, Deaton and Grandhi 2013). The results from these algorithms can then be built using AM technologies. Topology optimization methods have been used to design structures optimized to be minimally compliant at lower volume ratios and to optimize structures for best performance against a range of optimization targets. These include: the optimization of a structure with a multimaterial model (Jung and Gea 2006); for crashworthiness (Mayer et al. 1996); structures with multiple optimization objectives (Prasad and Emerson 1984); compliant structures (Bruns and Tortorelli 2003); structures with stiffness constraints (Chu et al. 1996); temperature reduction of heat conducting fields (Li et al. 2004); freely vibrating structures to maximise eigenfrequencies (Du and Olhoff 2007); and, a structure with a displacement constraint (Huang and Xie 2010). While not exhaustive, the above shows that topology optimization of structures has been applied to solve a wide range of objective functions.

Another of the applications of topology optimization methods includes their use to design energy absorbing structures (Yuge and Kikuchi 1995, Pedersen 2003, Jung and Gea 2006, Neves et al. 1995, Maute et al. 1998, Forsberg and Nilsson 2007, Huang et al. 2007, Kato et al. 2015, Zhang et al. 2016 and Wallin et al. 2016). These methods have been used for structures that utilised the technology's increased design freedom (Brackett et al. 2011). As well as the direct application of topology optimization methods, an indirect approach has included generating optimized unit cells which are then distributed throughout a structure using a metaheuristic optimization method. The intended result of this approach was to evenly distribute stresses throughout the loaded structure and then optimize the volume fraction of each unit cell using topology optimization to have a structure with material as evenly loaded as possible (Watts and Hague 2006). 
The task addressed in this research was to develop methods to optimize energy absorbing structures and to apply these techniques to a case study. These structures would need to absorb energy from an applied load with the greatest efficiency in terms of structural mass and to be manufacturable using SLM. As discussed above, topology optimization methods already exist, including for optimizing energy absorbing structures. For this research the Bi-Directional Evolutionary Structural Optimization (BESO) method was selected as the overall framework. It is worth mentioning that the focus of this research was on the development of algorithms to address constraints related to axisymmetric structures and additive manufacturing, rather than the BESO method. After each iteration the BESO method will select a subset of available elements as the current design for successive iterations. This is opposed to other methods, such as the SIMP method in which the design result is not a finite element mesh of discrete elements with a discrete binary value, but a mesh of elements attributed with continuous values. Comments and improvements on the binary methods can be found in (Rozvany 2009) and (Huang2010). Additionally, the bi-directional BESO method by adding and removing material between iterations is able to avoid settling on certain local optima that would be reached by solutions that only removed material between iterations. Of the three solutions to three different problems presented below it was the solution to the powder removal problem that made particular use of this property. The powder removal paths are adjusted at each iteration to the updated location of internal voids. Paths can be moved, removed or added and it would be unsuitable to use an optimization method that does not build back material once paths are moved or removed.

The case study on which this optimization method was applied was the design of a ring shaped structure that was required to absorb and contain projectile impacts on the inner surface of the ring. Using the BESO method unmodified would not have created axisymmetric or prismatic structures and would have led to the generation of a structure that had internal voids larger than what would be suitable for building using SLM. It would also not have allowed for the removal of metal powder from inside a structure after the build completed. These three problems and the methods and algorithms developed to address them are the focus of this paper. A further constraint was that the energy absorbing structure needed to successfully absorb the energy from the applied load without mechanical failure. This was addressed in previous work (Stojanov et al. 2016) in which a structural continuity constraint controlled to the volume ratio to ensure sufficient structural mass was present to absorb the energy from the load.

The first problem addressed was to design structures for which the location of the applied load was unknown. In the case study example discussed, the structure was to be designed for a single load the location of which, around the circumference, was unknown. The two other constraints related specifically to the SLM manufacturing process. The first of these concerned size limits on internal voids. There is a maximum size for any internal void of a structure built using SLM. Internal voids with overhangs over a certain length require additional support material during a build. Otherwise build failure can occur. There is previous work to control the maximum or minimum length of features in optimised structures. These often manage solid features in place of voids, but can often be swapped to operate on void regions. A common method is to use a penalty on the sensitivity values, examples include use with the homogenization or SIMP topology optimization methods (Haber et al. 1996, Brackett et al. 2011). In the latter case the penalty was applied to overhanging sections to reduce their presence in the final design, making them more suitable for manufacturing using SLM. Work also exists using the BESO method (Yang et al. 2002) and the method of moving asymptotes (Bruns and Tortorelli 2001) where a penalty value was used to reduce the total global perimeter of the structure. Other 
methods include a minimum size limit on the size of void regions in a mesh (Guest et al. 2004) that was later expanded to also include a maximum size on solid features (Guest 2009). The minimum only solution method required an additional filtering algorithm to filter sensitivity values and generate a discreet solid-void model from continuous values. The combined method required a series of cascading optimization solutions to meet added constraints, which, as with other solutions (Chen et al. 2008), leads to longer computation time. The minimum void algorithm is almost identical to the smoothing method used for BESO optimization (Huang et al. 2006). Similarly, a scan across the mesh has been used to ensure that all regions contained a certain proportion of solid or void elements (Alexandersen and Lazarov 2015, Poulsen 2003), having an effect similar to a penalty method. Methods that apply penalties or generalised filters such as smoothing operations used in image processing will apply a heuristic or penalty to direct the optimisation. The solution to be presented below will deterministically guarantee a maximum void size is respected and is fast to compute. The last constraint addressed relates to the removal of powder from inside a structure built using the SLM method specifically. Any internal void of a structure will be filled with unmelted metal powder at the end of the build. Similar to support structures, this material does not contribute to the mechanical performance, but does contribute to the structural mass. The solution presented is to generate interconnected links between voids to allow metal powder to be removed from inside a structure.

\section{Optimization task and optimization methods}

\subsection{Optimization task}

The optimization task was from a given design domain, loads and boundary conditions, to find a design that was optimized to absorb the energy from an applied load as efficiently as possible while meeting certain performance and engineering constraints, amongst which included the need that the structure were built using SLM. Here, efficiency is defined as the minimisation of structural mass necessary to absorb the necessary energy of the load. The BESO optimization method was used as a framework for the optimization method but additional algorithms were incorporated to address the issues discussed above. The BESO topology optimization method iteratively improves the design of a structure to try to reach an optimized result. This optimization method has been described in literature (Huang et al. 2006 and Huang and Xie 2007), including its previous use applied to the optimization of an energy absorbing structure (Huang et al. 2007).

The BESO method begins by taking the finite element mesh as the design domain of discrete 3D elements. The optimization method must select a subset of these elements to be the final design of the structure. Any design can be described by a vector $\mathbf{X}=\left(x_{1} \ldots x_{i} \ldots x_{n}\right)$, where $x_{i} \in\{0,1\}$. The values 0 or 1 designate that element $x_{i}$ is absent or present in the design. A function, $f(\mathbf{X})$, maps from designs to their mass and the focus of this research is the search for a design, $\mathbf{X}$, that minimises $f(\mathbf{X})$ while also meeting all other constraints. For the optimization method used to improve the design of an energy absorbing structure, i.e., the objective function $f(\mathbf{X})$ becomes the mass of the structure, the equation used to assign a sensitivity value to elements is (Huang et al. 2007)

$$
\alpha_{i}=\frac{V_{i}}{V}-\frac{E_{i}}{E}
$$

where $V$ and $V_{i}$ are the total volume of the structure and the volume of element $i$, and $E$ and $E_{i}$ are the plastic energy of the structure and element $i$. The formula to calculate $E_{i}$ is as follows 


$$
E_{i}=\int_{\text {element } i}\left(\int_{0}^{\varepsilon_{j k}^{p}} \sigma_{j k} d \varepsilon_{j k}^{p}\right) d V
$$

Where $\sigma_{j k}$ and $\varepsilon_{j k}^{p}$ are respectively the stress and plastic strain components and the convention of Einstein summarization for repeated subscripts is applied in Eq. (2). The plastic energy of an element is denoted as variable ELPD in the commercial software Abaqus, which was used in this study. The sensitivity value $\alpha_{i}$ measures the sensitivity of the objective function to the addition/removal of that element. During each iteration of the optimization process the volume ratio, $\Psi$, of the structure is lowered to generate an optimized structure or raised when necessary to meet constraints. Sensitivity values are used to prioritise the addition/removal of elements to maintain an optimized structure.

A necessity for an energy absorbing structure is that it properly absorbs the energy of a load and does not fail. Here a fracture between critical regions of the structure will correspond to a failure of that structure. An energy absorbing structure will need to absorb the energy of the applied load, $\Theta$, as a performance requirement. Given loads and boundary conditions a structure can absorb $\Theta_{\max }$ energy before fracturing. For a successful structure $\Theta_{\max }>\Theta$ and $\Theta_{\max }<\Theta$ for a failed structure. This constraint is determined indirectly. The procedure and its integration into the framework of the BESO method is available in the literature (Stojanov et al. 2016).

The other constraints were addressed in the work presented below. The first was to ensure that the design generated be axisymmetric. For the case study the structure was a cylindrical containment ring which absorbed the impact from a rotor segment. The impact location around the circumference was unknown and so the final design had to perform equally well irrespective of the circumferential position of the rotor impact. An axisymmetric design was required and so this constraint needed to be imposed on the optimization method. It is noted here and expanded on below that the solution to impose the axisymmetric condition on a thin walled axisymmetric structure is similar to generating a prism with a uniform cross-section along the longitudinal direction and as such the one solution presented below is equally applicable to either case. The method to address this constraint is described in Section 2.2. A function $s(\mathbf{X})$ mapping to $\{0,1\}$ is said to describe whether a structure is (mapping to 1 ) or is not symmetric or axisymmetric as required.

The second constraint was that the length across the largest internal void of a design, $L_{L}$, was below a maximum limit, $L_{m}$. The value $L_{m}$ will vary according to the individual circumstances present for the designer, such as the machine and material used during the SLM process, but is a definite limit on the size of internal voids that can be built. Internal voids above this limit will lead to build failures and an unsuitable design. The optimization method needed to produce designs that respected definite limits on the maximum size of voids and the minimum distance between voids in order that problematic features were not generated. The method to address this constraint is described in Section 2.3.

Further than the size of internal voids was the requirement that there was an interconnected path from each void to the outside of the structure. This is a necessary feature of all structures built using SLM. All internal voids are filled with metal powder during construction and this powder, while contributing to the overall structural mass, provides no mechanical benefit. If a function $g(\mathbf{X})$ maps from a design to the set $\{0,1\}$, where 1 corresponds to a design in which a structure contains no voids, or voids are interconnected, and 0 is a structure with internal voids without a path through which unsintered powder can be removed, then the final constraint was that $g(\mathbf{X})=1$ for the resulting 
design. For ease of discussion, voids with a connected path will still be referred to as internal to the structure despite the presence of connecting links to the outside of the structure. The method to address this constraint is described in Section 2.4.

The optimization requirement was then to

$$
\begin{aligned}
& \text { minimise } \\
& \qquad \begin{array}{c}
f(\mathbf{X}) \\
\text { subject to } \\
\Theta_{\max }>\Theta ; \\
s(\mathbf{X})=1 ; \\
L_{L}<L_{m} ; \\
g(\mathbf{X})=1 .
\end{array}
\end{aligned}
$$

\subsection{Method to force an axisymmetric design}

As with other topology optimization methods, when given a model, including loads and boundary conditions, the BESO method will search for an optimized structure that best meets those particular conditions. The same geometry with different conditions will produce a different result and these two results may be particularly unsuitable for use under the other's loads and boundary conditions. This is illustrated in Fig. 1 where a simple model is optimized twice to produce two very different solutions. The left and right side show an otherwise identical optimization with the only change in the location of applied loads and boundaries. The structures when optimized to maximise strain energy density show very different results where each structure would be far less suitable when applied to the other's loads and boundary conditions. The design case addressed here was one for which the location of applied loads along the circumference was unknown and so the resulting structure needed to be suitable for a load applied at any location along this circumference.

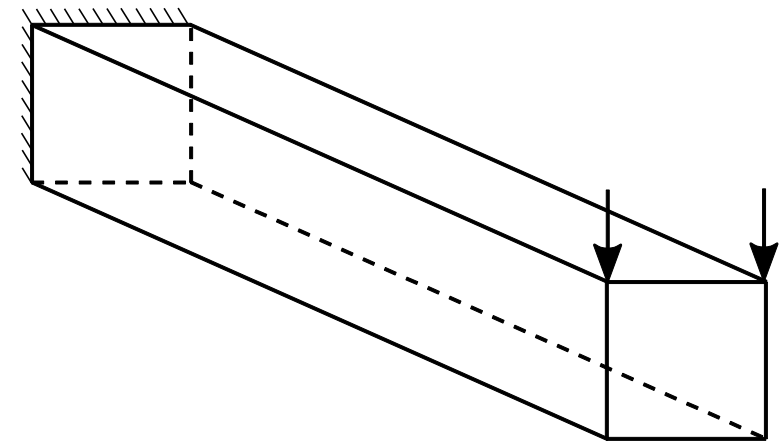

i. Case with built up end and two point loads at far end.

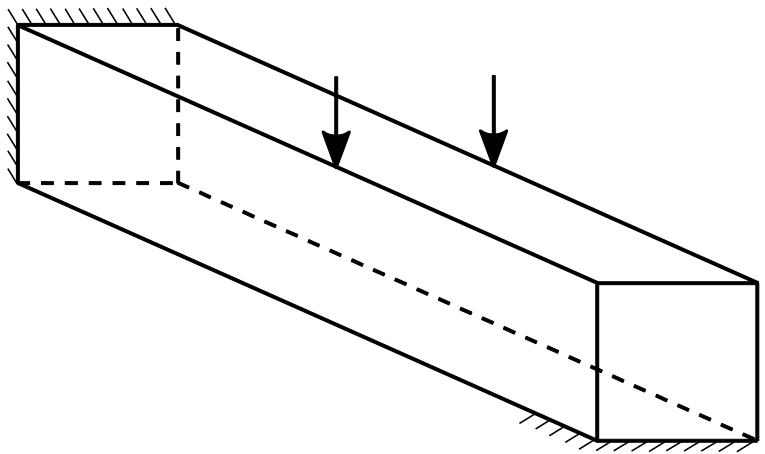

ii. Case with built up end with two point loads in the centre and additional support at the far end.

(a) Diagram of design domain of the bar with all boundaries and loads. The built up end is fixed in displacements and rotations.
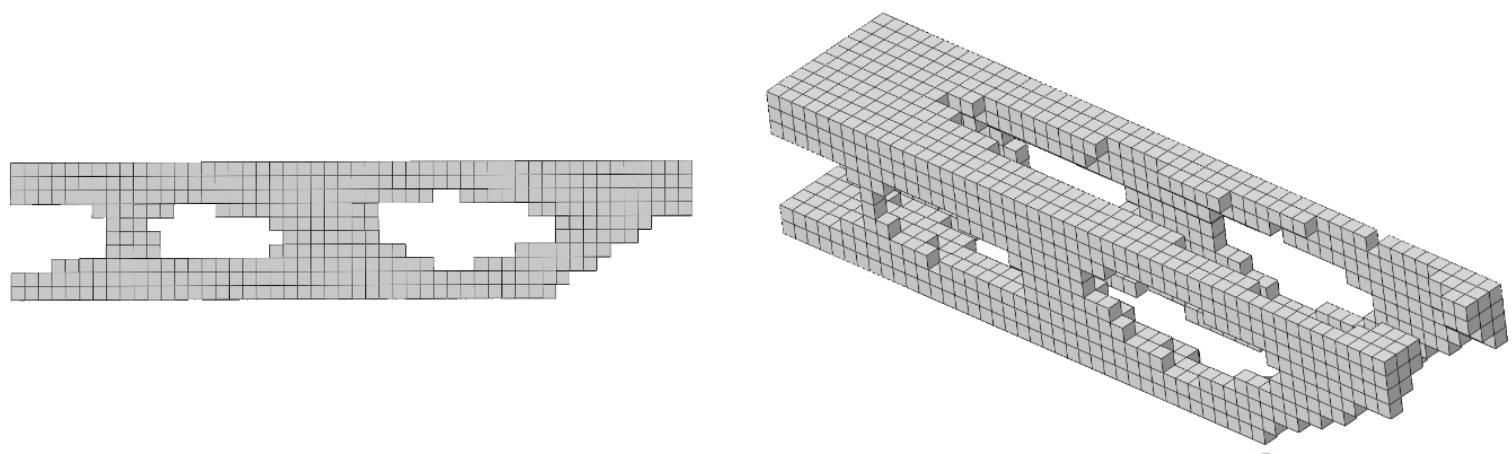


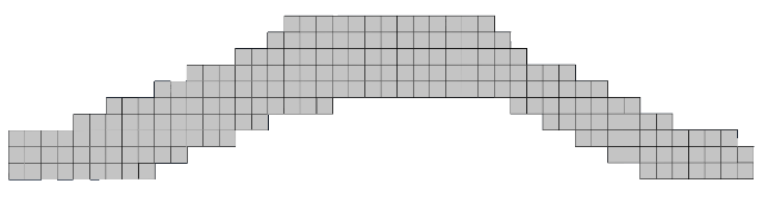

i. Side view

(c) Result for load at the centre, supports at both ends.

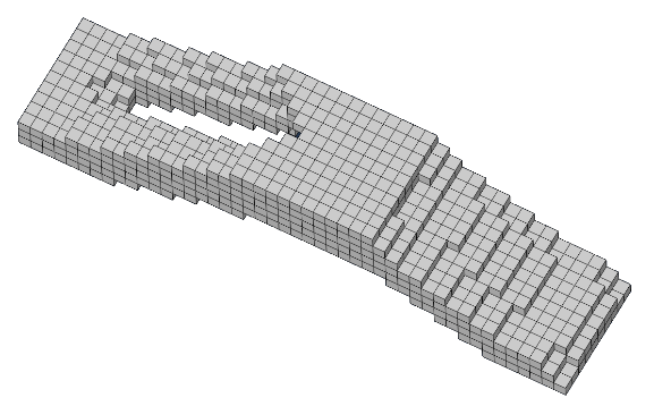

ii. Orthographic view

Fig. 1 Simple bar examples with different loading location and boundary leading to different non-uniform cross-section designs

There is a solution to problems similar to the above: a solution to find a compromise between multiple load cases, using the BESO method, is available in the literature (Querin et al. 2000). Here a compromise solution is produced by analysing multiple load cases and taking average sensitivity values. In the case for the containment ring there is a load case for every possible impact location around the circumference. An Individual finite element analysis for each load case around the circumference is not a realistic solution.

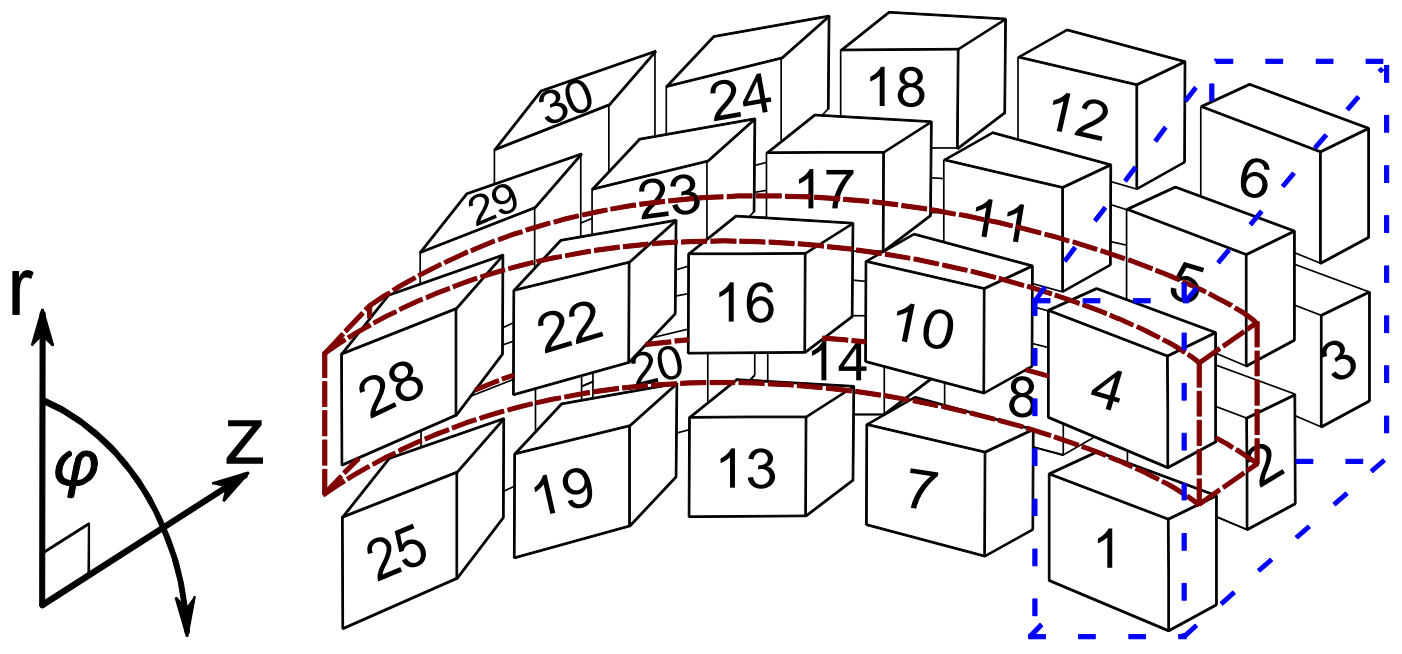

Fig. 2 Illustration of the element grouping method along the circumferential direction to force an axisymmetric design

The method used for this research began with the user selecting the cross section of the thin walled axisymmetric structure. An illustrative example is shown in Fig. 2 with the elements in a thin-walled axisymmetric geometry highlighted with a blue/sparsely-dashed line. Using only this information all elements in the same relative position in each cross section can be determined. One such collection of elements is shown highlighted by a brown/closely-dashed line in Fig. 2. Once grouped, element sensitivity values were averaged to arrive at an average sensitivity value for each group of elements. A volume weighted average scheme was used for the case study however the mesh was regular between elements and as such an arithmetic average value was also suitable. Hexahedral elements were used for this case study, though with any element shape that provided regular cross sections suitable for use with this method. Rather than adding/removing individual elements using the BESO 
optimization method, elements were added/removed by groups, prioritised using their average sensitivity values.

An overview of this process is

1. The designer selected the elements in the cross section of the structure.

2. The algorithm grouped elements at the same position in the structure's cross section.

3. Element sensitivity numbers were averaged before ranking and added/removed from the design as a group.

The above process allowed for the BESO method to otherwise operate as usual while guaranteeing axisymmetric structures that were still optimized to perform as efficiently as possible given this constraint. The method to force an axisymmetric design, as illustrated by Fig. 2, can be equally applied to force a design of a prismatic component with uniform cross-section when the loading location is unknown, as discussed in Fig. 1.

\subsection{Method to control the size of voids in topology optimized structures}

Structural topology optimization creates voids inside the structure that it is optimizing. An optimized design with large internal voids cannot be built successfully using SLM without any additional support material as overhangs over a certain length will distort or lead to build failure during the manufacturing process. The threshold before which supports are required may vary depending on the material and the particular machine used. For example, the experience of the Monash Centre for Additive Manufacturing at Monash University is approximately $5 \mathrm{~mm}$ for Nickel superalloy powders (Jarvis, 2013). Depending on the shape of a void, the maximum overhang length can be any side of the void perpendicular the SLM building direction. Considering this, the maximum overhang length is referred to the maximum length of a void in the building direction in the design optimization.

The method developed controlled both the size of internal voids and the distance between voids, ensuring that no voids of length greater than $L_{m}$ were created. The method mapped a finite element mesh to a graph representing the relationship of neighbouring elements. Adjacent finite elements were represented by graph nodes connected by an edge if these elements were defined with common finite element nodes. By searching this graph it was always possible to find all voids in the mesh. By considering only deactivated element, voids will be isolated components of nodes on that graph.

To map between $L_{m}$ and a length scale relevant to the finite element mesh is straightforward using

$$
n_{v}=\left\lfloor\frac{L_{m}}{L_{e}}\right\rfloor .
$$

Here $L_{e}$ was the length of the longest edge of an element and $n_{v}$ then becomes the maximum number of finite elements that will be allowed in a void. In this way the sizes of voids were independent of the mesh and depended on the parameter $L_{m}$ provided. A similar method can be used for $n_{b}$, the minimum distance between voids. For the case study described in this paper the values of $n_{v}=6$ and $n_{b}=2$ were used.

When the BESO method was removing elements from the mesh in order to decrease the value of $\Psi$, the mesh was processed. The process found all elements in voids by searching for connected components. All components were also searched to find all neighbours of order $n_{b}$ near to other voids. If nearby voids were found, this combined group of elements was classified as a void of a size equal to 
the sum of both voids and $n_{b}$. If voids reached the limit of $n_{v}$, all neighbours to these elements of order $n_{b}$ were flagged as unavailable for removal until the next time the mesh was processed. This enforced a strict upper limit on the size of voids and a minimum distance between voids. Allaire et al. (2016) applied a level set method to control the local thickness in structural optimization.

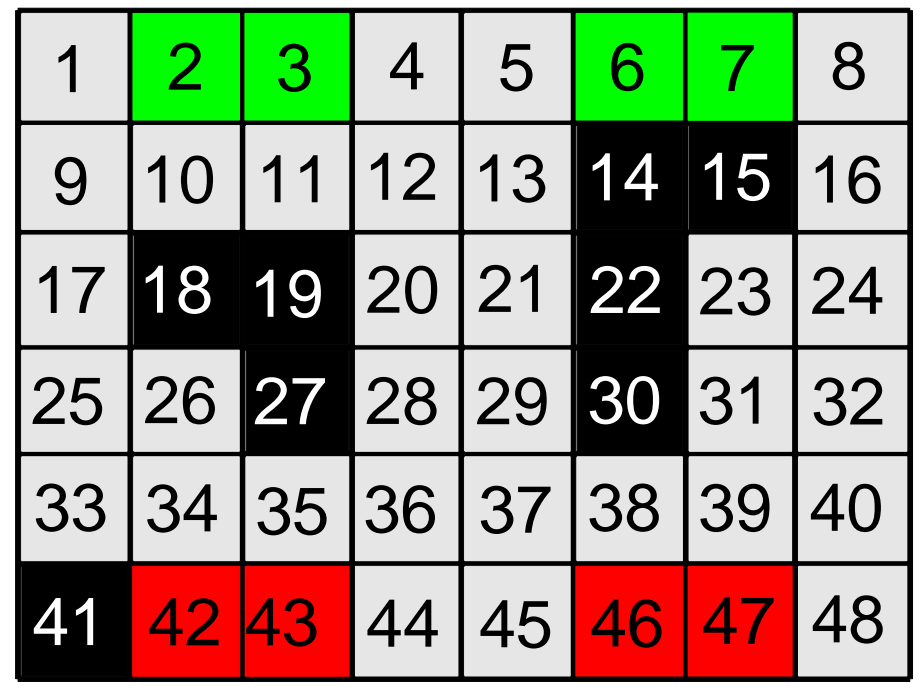

Element selected as part of an entrance group.

Element selected as part of an entrance group.

Element removed from current design. (Void element)

Element included in current design. (Solid element)

Fig. 3 Illustrative example of a cross section with voids. Entrance/exit elements shown in green and red

A 2D illustrative example is shown in Fig. 3. Voids are shown as black shaded elements. Given $n_{v}=4$ the void comprising elements $\{14,15,22,30\}$ could not be enlarged using this process, all neighbours of order $n_{b}$ or less are barred from removal. Elements $\{18,19,27\}$ are smaller than this limit. Nearby neighbours might be removed if they had suitable sensitivity values, but not if they are within distance $n_{b}$ of other voids.

The procedure then is

1. The analyst specified the parameters $n_{b}$ and $n_{v}$.

2. All voids with $n_{v}$ elements were identified before material was removed by the optimization method.

3. All neighbours of order $n_{b}$ or less were identified.

4. These identified elements were barred from being removed.

5. Steps 2 to 4 were repeated each time the design would be changed by the removal of any further material.

It is noted that this bottom-up approach is different to those reviewed earlier which might employ a penalty method. Such methods cannot be guaranteed to provide a definite limit while this method guarantees. Further, searching for components in a graph completes in linear time, providing a computationally simple process to controlling void sizes in an optimized structure. The optimization still works on sensitivity values calculated using the BESO optimization method, or on element groups 
using the method described in Section 2.2. Advantages of this include the ability to apply this constraint on any other optimization objective to which BESO can be applied. The binary nature of the BESO method allows for easily measuring void sizes and determining how to process them without intermediate steps to map from a continuous field to a discreet structure.

\subsection{Method to generate a path to remove powder for components built using SLM}

The other constraint when optimizing a structure that will be manufactured using SLM is that any internal voids in a design will be left filled with powder after manufacturing. Removing this powder from the structure requires that there is a path from the outside to the void. It is necessary that these connections:

- Have the smallest total length, disturbing the design of the structure as little as possible.

- Connect to the outside of the structure, ensuring that the path is available for the powder to be removed.

- When generated will still lead to a structure that avoids catastrophic failure and that this can be verified during the optimization process.

The analyst was required to provide one or more pairs of sets of entrance and exit elements. These were elements from which the connecting path would enter and exit the structure. There is no meaningful difference between entrance/exit except that element sets come in pairs. It is noted that the process to be described requires the analyst to provide only this information and is otherwise completely automated and mechanically computable.

The process was performed along the following steps. The first two described in Section 2.4.1 and the next two in Section 2.4.2:

- Voids were grouped into collections according to their nearest entrance/exit pair.

- Finding the shortest path connecting all voids in a collection.

- Translating links along the circumferential direction.

- Generating links by connecting elements.

\subsubsection{Algorithms to group voids to an entrance/exit pair and to find the shortest path connecting voids}

An arbitrary number greater than or equal to one pair of entrance/exit element sets were provided by the analyst. Each void was identified by finding components using the same process described in Section 2.3. Once identified voids were grouped to the nearest entrance/exit element set using the following:

1. Each entrance/exit pair had a centroid calculated for both element sets in the pair. $C_{n_{j}}$ and $C_{x_{j}}$ are the centroids for the $j$ th entrance/exit pair.

2. For each pair $C_{n_{j}}$ and $C_{x_{j}}$ an average position $C_{s_{j}}$ was calculated. This was a middle position for the entrance/exit pair of element sets.

3. For each void an average centroid position $C_{v_{j}}$ was calculated. This was a centroid calculated from the volume that included all elements in void $j$.

4. For $j$ entrance/exit pairs, voids are assigned to the pair for which $\left\|C_{v}-C_{S_{j}}\right\|$ is the smallest. 
The task was then that for each grouping of a pair of entrance/exits and nearby voids needed to be connected by an open path through which metal powder remaining after the SLM manufacturing process could be removed. Ideally, such a path would be as short as possible in order to change the design as little as possible. For that reason the shortest path was found which began at one end of the entrance/exit pair, visited all voids, then exited from the other end of the entrance/exit pair. The "brute force" method completes in factorial time, however efficient solutions are available in the literature (Lin and Kernighan 1973).

\subsubsection{Algorithm to translate the links between voids}

The method as described above was applied to the case study at a particular cross section. All of the voids ran through the circumference of the ring. The shortest links would therefore be links that ran across cross sections between design voids. A single cross section with all links would generate a single point of greatest weakness. Links were therefore distributed evenly along the circumference. For a segment of an axisymmetric structure of, $m$, elements along the circumference with, $n$, links each link was placed evenly along the circumference. Each link was generated at a period, $t$, of elements along the circumference between links. This parameter was found using

elements for a segment and

$$
t=\left\lfloor\frac{m}{n+1}\right\rfloor
$$

$$
t=\left\lfloor\frac{m}{n}\right\rfloor
$$

for an axisymmetric structure that completed full ring instead of a small segment. Links between any two voids were generated by removing all elements intersected by a line from the centroids of the two nearest elements in each of the two voids. 


\subsection{Incorporating void control and connectivity in the overall optimization}

process

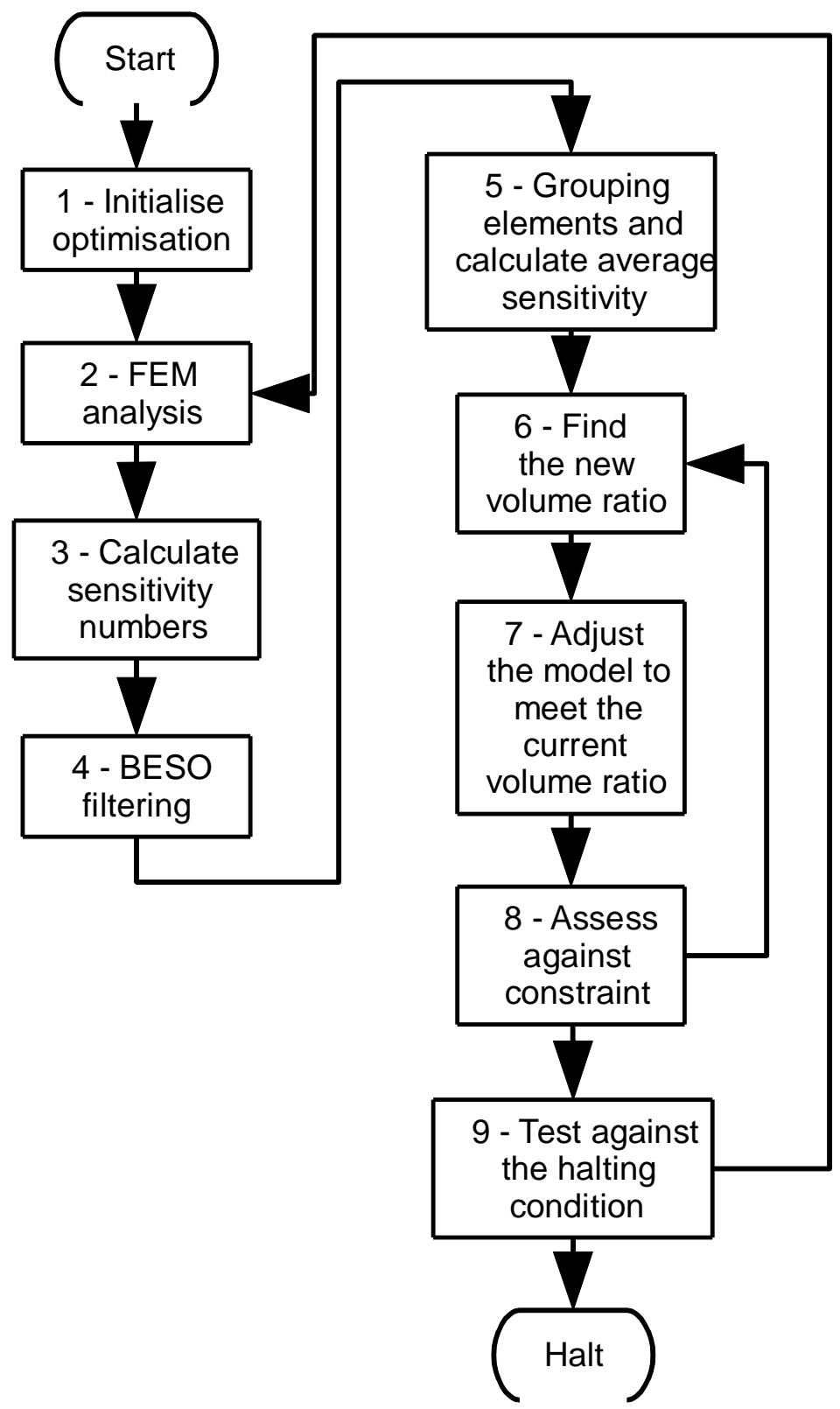

Fig. 4 High level flow chart of the optimization process

The sequence in Fig. gives a high level overview of the BESO optimization method and how it was implemented in this research together with the constraints described in Section 2.1. The methods described in Section 2.2 correspond to step 5 of Fig., the methods in Sections 2.3 and 2.4 correspond to step 7 and methods previously published correspond to steps 8 and 9 . 

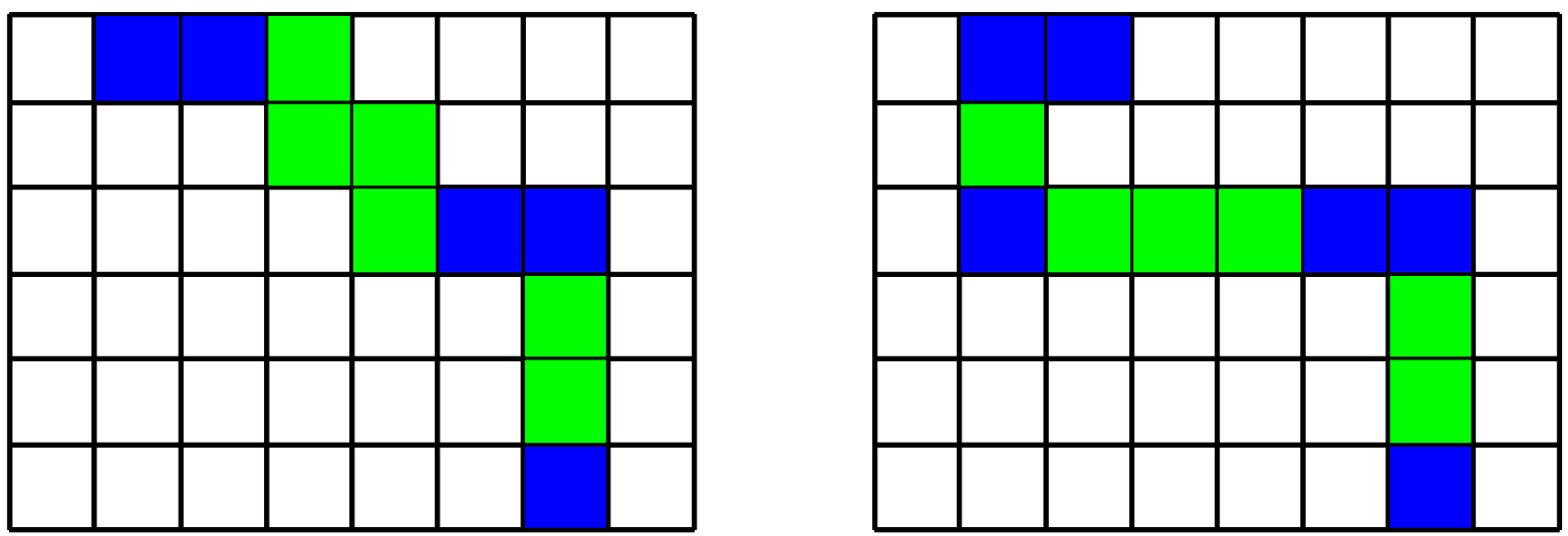

Fig. 5 Example of the rerouted paths before (left) and after (right) a new void is created. Blue elements are voids with green elements a connecting path between voids

New voids created by a reduction of the volume ratio of the structure could change the shortest path between voids. An illustrative example is shown in Fig. in which a new element removed has caused a change in the shortest path. Each change in the model caused by the optimisation method needed to be followed by a repeat of the void control and void connectivity algorithms to adjust to the new changes in the geometry. Further, these links could affect the strength and energy absorption performance of the structure. The continuity constraint ensures that the structure is sufficiently strong for the given load. The structure must be checked after these links are incorporated. Checking after links are made ensures the design remains suitable to absorb the energy from the loads even after these links have been generated.

\section{Application to a case study}

\subsection{Problem description}

The case study focused on optimizing the design of an energy absorbing metal ring surrounding a disc that bursts into three equally sized segments. An example of such rings is found around turbine and compressor fans in aircraft in which failure of turbines needs to be contained to protect surrounding equipment. The ring must keep all fragments inside without allowing any to perforate through or to cause the ring to open (failure due to hoop stresses). The mechanism through which this energy is absorbed and removed from the system is through the plastic deformation and local damage of the containing structure. The objective of the case study is to design a new containment ring with reduced weight, which still maintains its performance to absorb the same amount of given energy. A model of the ring with the three segments is shown in Fig.. The symmetries are labelled $S_{n}$ and $S_{n}^{\prime}$. For design and reasons of economy the ideal structure would perform these tasks using as little structural mass as possible. The nickel alloys INC 716 and INC 625 were chosen for the projectile and the ring in the optimization design of the containment ring. The material data of these two materials can be found in the following publications (Kobayashi et al. 2008), (Pereira and Lerch 2001), (Haynes International Inc. 2001), (Special Metals Corporation 2006), (ASM International 1967), and (Samanta et al. 2012). 


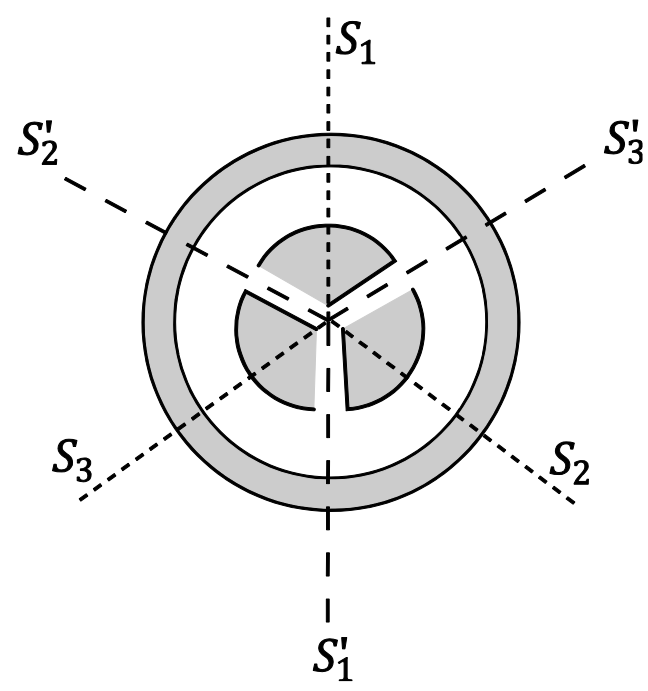

Fig. 6 A diagram of the symmetry in the fragment-ring interaction for rotor segments of $120^{\circ}$

\subsection{Finite element model}

Before an optimization analysis of a structure can begin, it is necessary to create a finite element simulation of the model and the loads to which it is subjected. As shown in Fig. 6 the interaction featured symmetry allowing full results to be obtained using a fraction of the complete ring. Only $1 / 6$ of the circumference was required as the simulation was symmetric across all of the dotted lines in Fig.6. The use of $1 / 3$ brought a sufficient saving in computation time. The final result would be close to, but not entirely axisymmetric. The presence of the small connecting paths at certain locations results in a structure that is not precisely axisymmetric. The use of a $1 / 3$ model allowed the final results to repeat evenly 3 times around the circumference. A diagram of the 1/3 model is shown in Fig. 7 . Further symmetry existed because the fragment was assumed to impact at the centre along the height of the ring and was therefore symmetric above and below the centre of the ring. This was also used when modelling to improve computation time. The finite element model had symmetric boundary conditions applied to the three edges across which symmetry existed with one edge free of symmetric boundary conditions. This free edge is the one on the right of the two visible in Fig.. In this case study, the projectile was assumed as linear elastic with the Young's modulus of 205 GPa and Poisson's ratio of 0.3 . The projectile has a radius of $38 \mathrm{~mm}$ and a thickness of $24 \mathrm{~mm}$. General contact was used with the outer surface of the projectile and all internal and exterior element surfaces of the ring. Abaqus/Explicit was used for the finite element analysis. This was motivated by material and geometric non-linearities and a total simulation time of less than 0.01 seconds for all interactions to complete. A convergence study was completed on the mesh used in the finite element model in the case study. A mesh size was selected that was finer than the point at which the energy absorbed by the structure began to converge. This gave models that took approximately between 50-60 minutes to simulate in Abaqus/Explicit using a single core on a desktop computer and a further 3-5 minutes to process the rest of the optimization steps before the next iteration. As the optimization calculations took much less time to process than the finite element simulation no further efforts were made toward efficiency of the optimization software. 


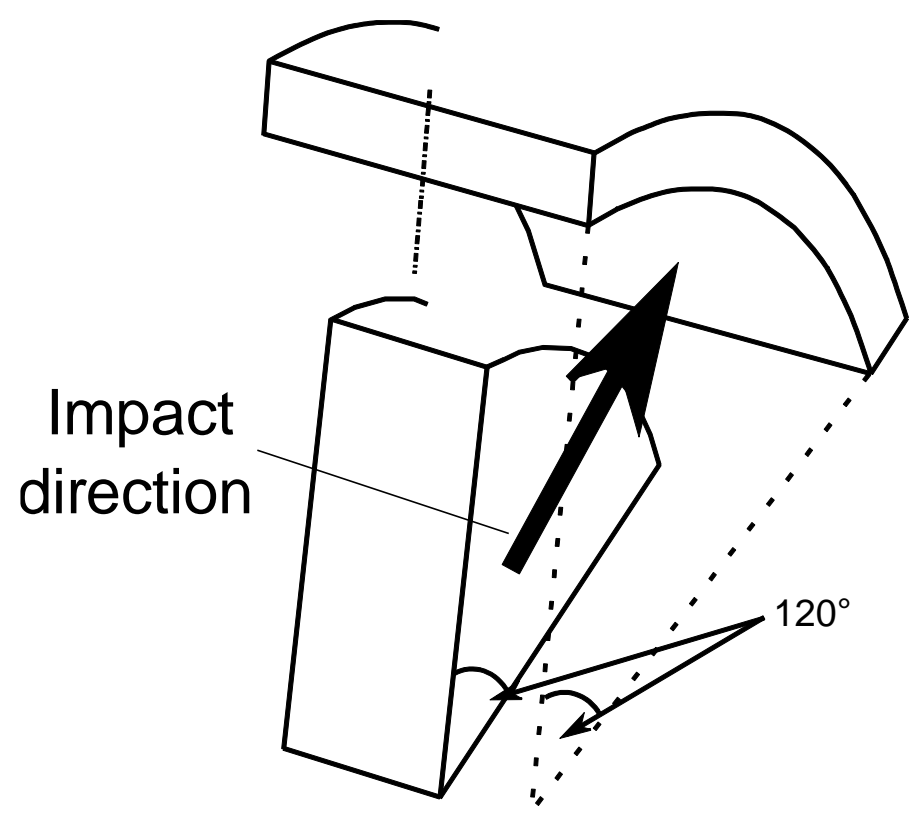

Fig. 7 Assembly of the finite element model used to simulate the interaction between the ring and projectile

\subsection{Results and discussion}

There were two optimization analyses performed on the ring. Both used the same BESO method but varied in the use of the void control scheme described above. These will be referred to as Scheme 1 (the model optimized without the void control scheme) and Scheme 2 (with the void control scheme).

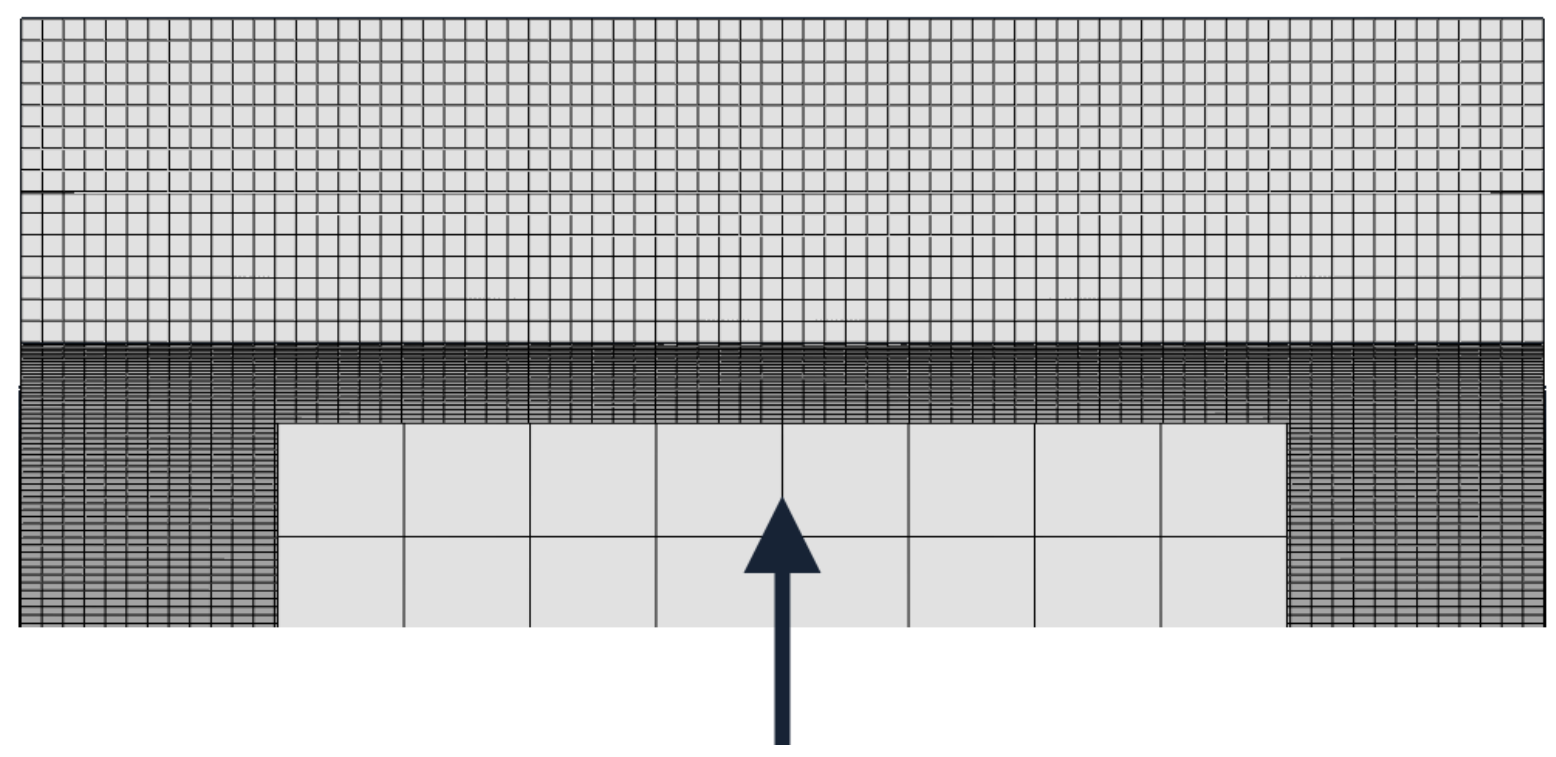

(a): Original cross section before optimization shown with the projectile impacting on the inner surface of the ring. The direction of the projectile's motion is shown with the arrow.

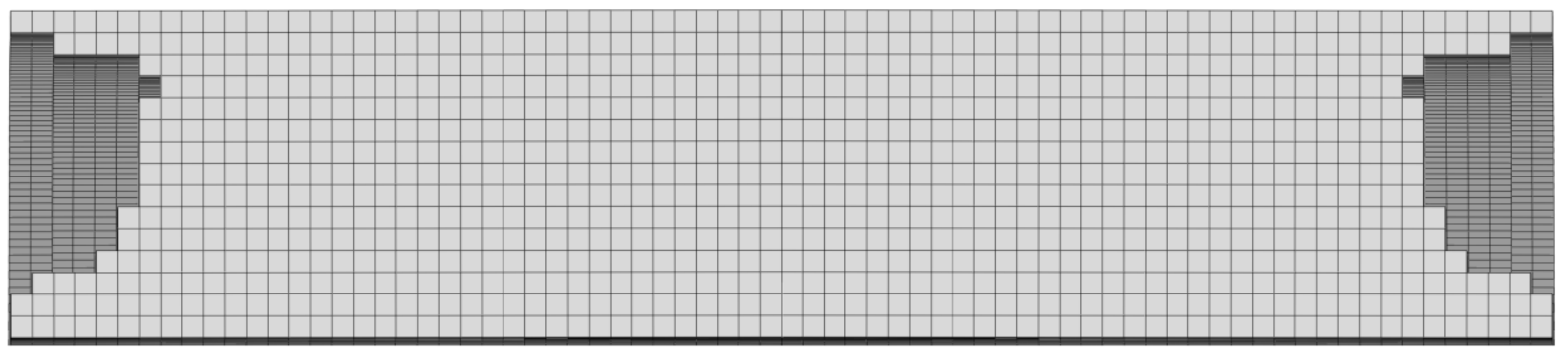




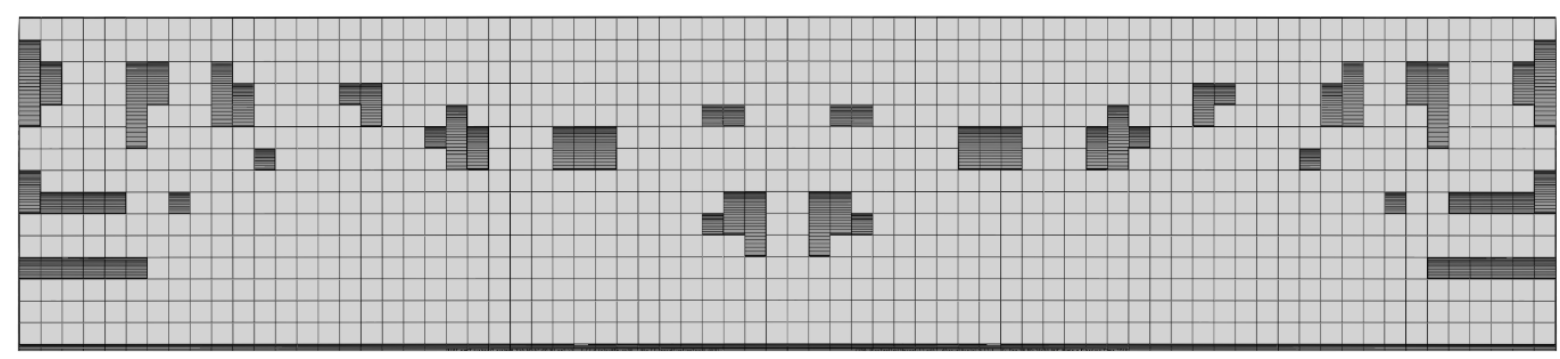

(c): Result using Scheme 2. The structure has $89.9 \%$ of the mass of the original structure $(10.1 \%$ removed).

Fig. 8 Cross section of final results for the two optimization schemes. Comparison made against the entire design domain

The results for the two schemes were designs that were successful in containing the impactor within the containment ring without failure of the containment ring structure. The optimization removed $11.2 \%$ of the mass of the original structure when Scheme 1 was used and removed $10.1 \%$ for Scheme 2. At lower volume ratios, Scheme 1 results contained internal voids at the centre of the cross section. Again, this matched Scheme 2 results with most material removed from the left and right ends of the cross section, and slightly more material removed from the centre.
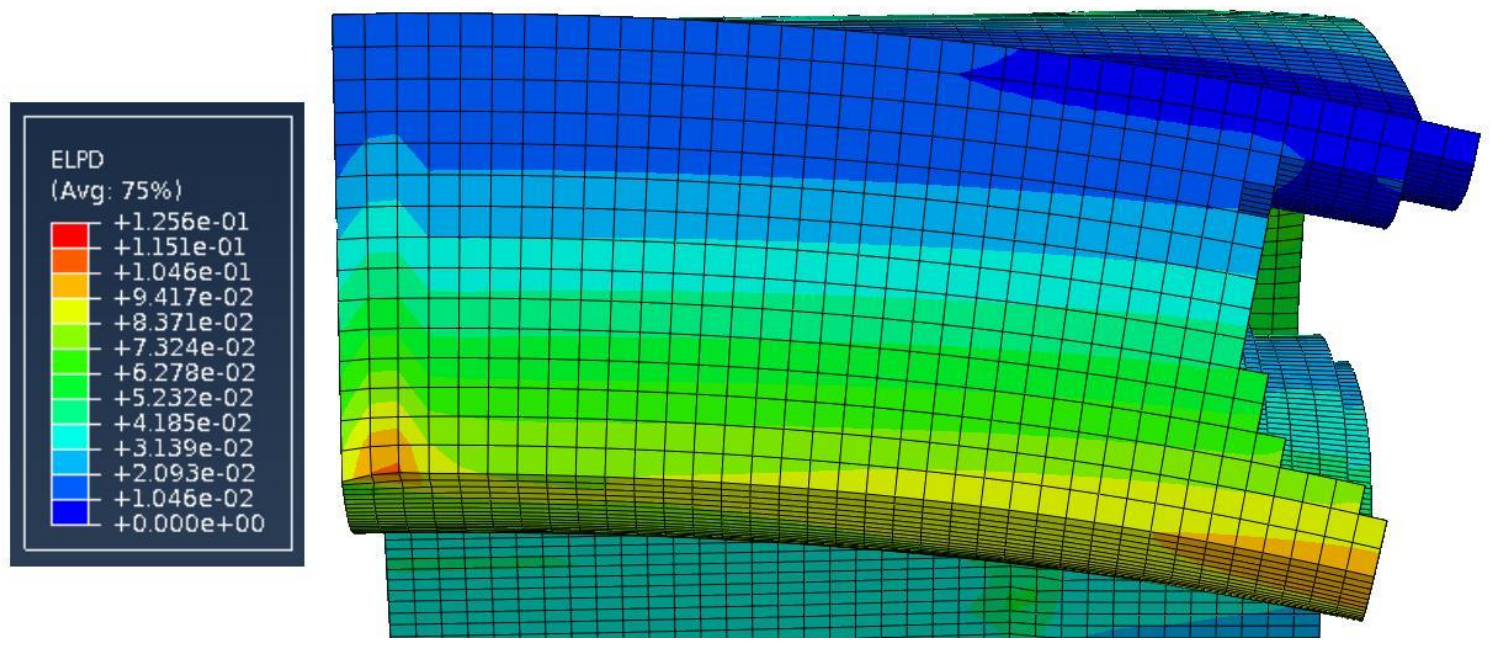

Fig. 9 Displaced cross section of the containment ring showing bands of plastic energy absorption. The geometry is the resulting geometry using optimization Scheme 1 shown in Fig.(b). The cross section shown is at location distant, circumferentially, from the impact location. (Contours indicate increasing amounts of plastic energy absorbed in the downward direction of the cross section)

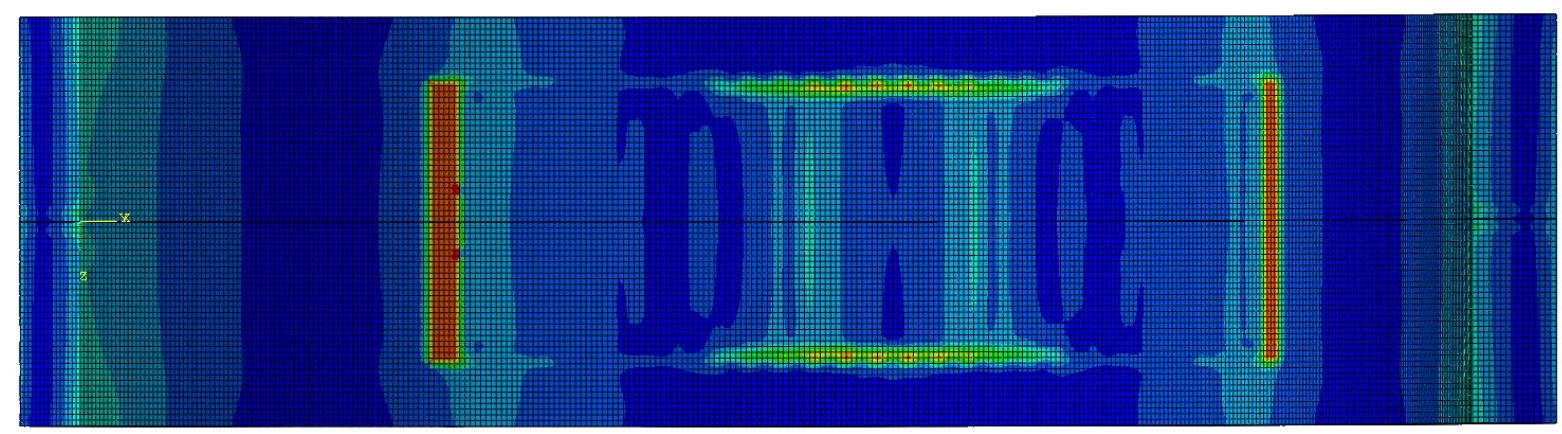

(a): View of the ring inner surface. 


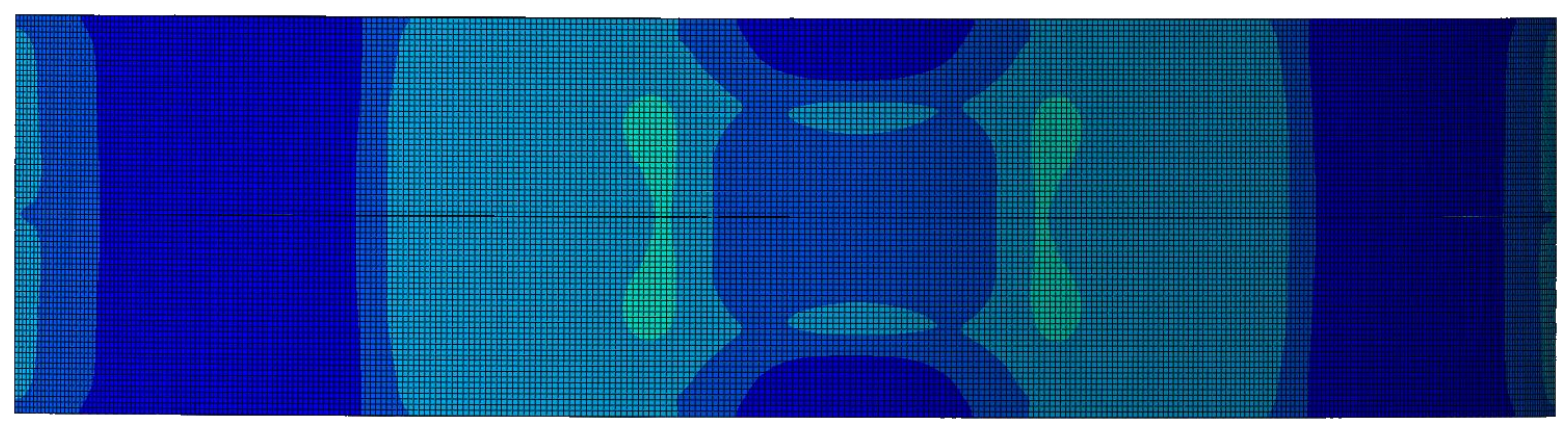

(b): View of the ring outer surface.

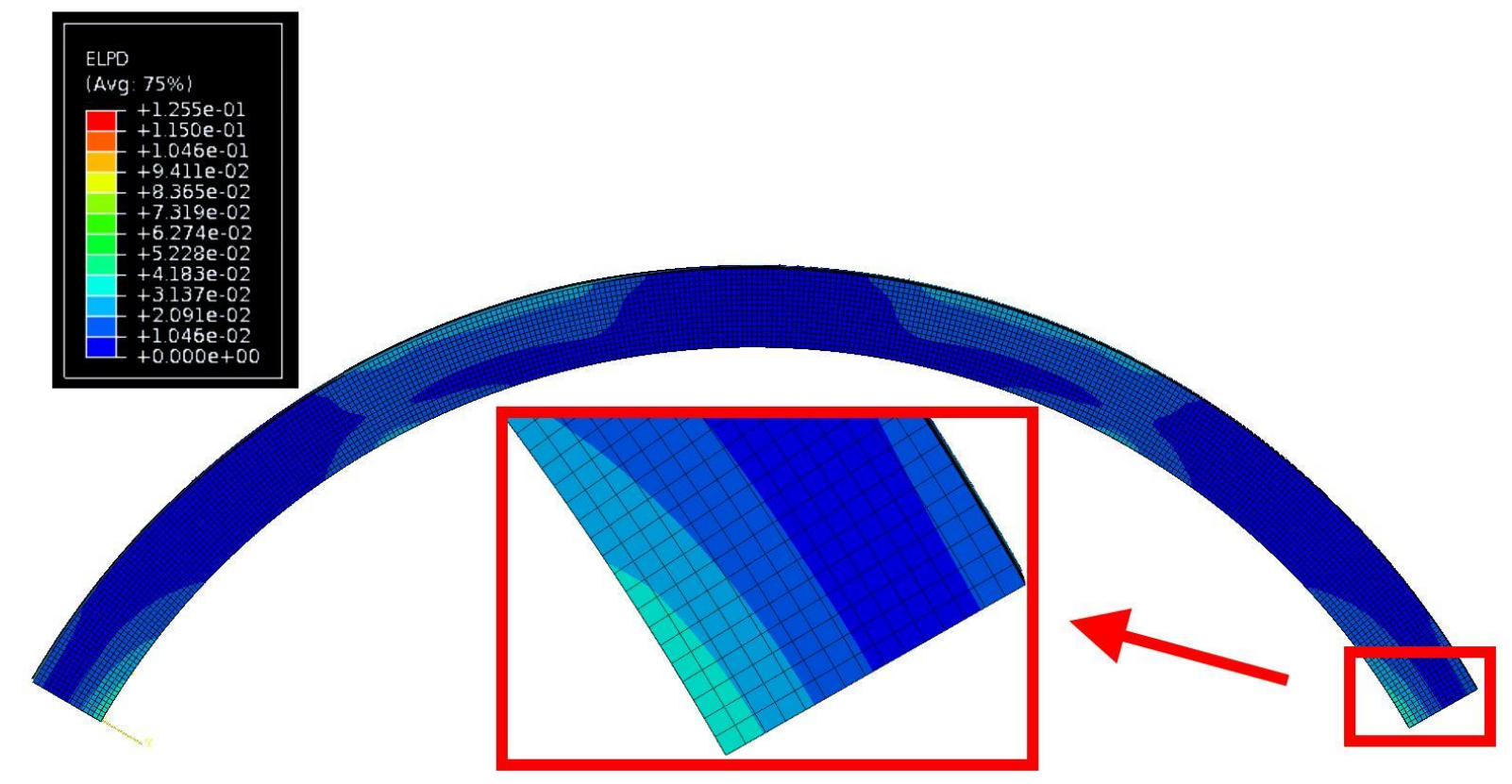

(c): Side view.

Fig. 10 Distribution of plastic strain energy in the containment ring segment, before optimization, from the finite element simulation. (Colour scheme as in Fig.)

\subsubsection{Axisymmetric designs}

The results in Fig. show that the structure generated is of an axisymmetric design. The cross section generated when no void management options were selected, shown in Fig.(b), has the same crosssection all along the circumferential direction. The same is the case for the void controlled structure in Fig.(c) except for those cross sections in which interconnecting links have been created. Fig. shows the paths of two such links, marked with a dotted line. There is only one link at any cross section, leading to a minimum variation from a completely axisymmetric design.

It is important to note that a model of fragment-ring interactions proposed by Hagg and Sankey (1974) assumed that there were two important responses from the containment ring due to the rotor's impact. The first of these were the rotor's local interaction with the containment ring. Here local deformation of the ring includes shearing of the ring material around the perimeter of the rotor and compression of the ring immediately in front of the impacting rotor. The finite element results showed this as well as a significant amount of plastic deformation on the outer surface of the ring, near the impact location, as it was deformed in tension while the ring diameter was enlarged. The Hagg and 
Sankey model also describes a global response due to hoop stresses generated in the ring. In the finite element model, at the far ends of the ring segment, there is a linear gradient in the tension caused by these hoop stresses. The inner surface experienced a greater load than the top. This is shown in Fig. and Fig. 10(c). Therefore, when viewed overall the result is that the inner and outer surfaces experienced larger plastic deformations than the centre, with the inner being the greater of the two. The results in Fig.(a) matches this expectation with the resulting design showing a cross section that tapers in the centre, with the inner surface thicker than the outer surface. Before the analysis the top surface was selected as unavailable for removal. The taper does exist because there was still some plastic deformation on the top surface and to provide a smoother taper and avoid stress concentrations.

\subsubsection{Void control}

The results in Fig.(c) show that, in contrast to Fig.(b), voids created are a maximum size of 6 elements, with a minimum of 2 elements between full sized voids. This is the result expected given the values of $n_{v}=6$ and $n_{b}=2$ used for this analysis. Of further interest is to see that the cross section shown between the two different optimization schemes in Fig.(b) and (c) shares similar characteristics, but varies according to the design features selected for the optimization. The far ends feature empty space for Scheme 1 and a larger number of voids for Scheme 2. Further, the pre-optimized loads in Fig. 10 show that the greatest loads are on the inner and outer surfaces. The resulting geometry for Scheme 1 left more material near the surfaces at the far ends. Similarly, there is a line of voids slightly higher than the centre of the cross section. Consistent with analysis results, including that the inner surface tends to absorb more energy than the outer.

There are shear loads on the containment ring around the perimeter of the impact location of the rotor onto the ring. These form an outline around the projectile visible in Fig. 10(a) where these increased loads are visualised as increased plastic deformation. This is accommodated in the optimized geometry in Fig.(c) with the line of voids raised around the points corresponding increased shear loads along the bottom surface. This leaves the bottom surfaces around these shear loads with more solid material. 
3.3.3. Void connectivity

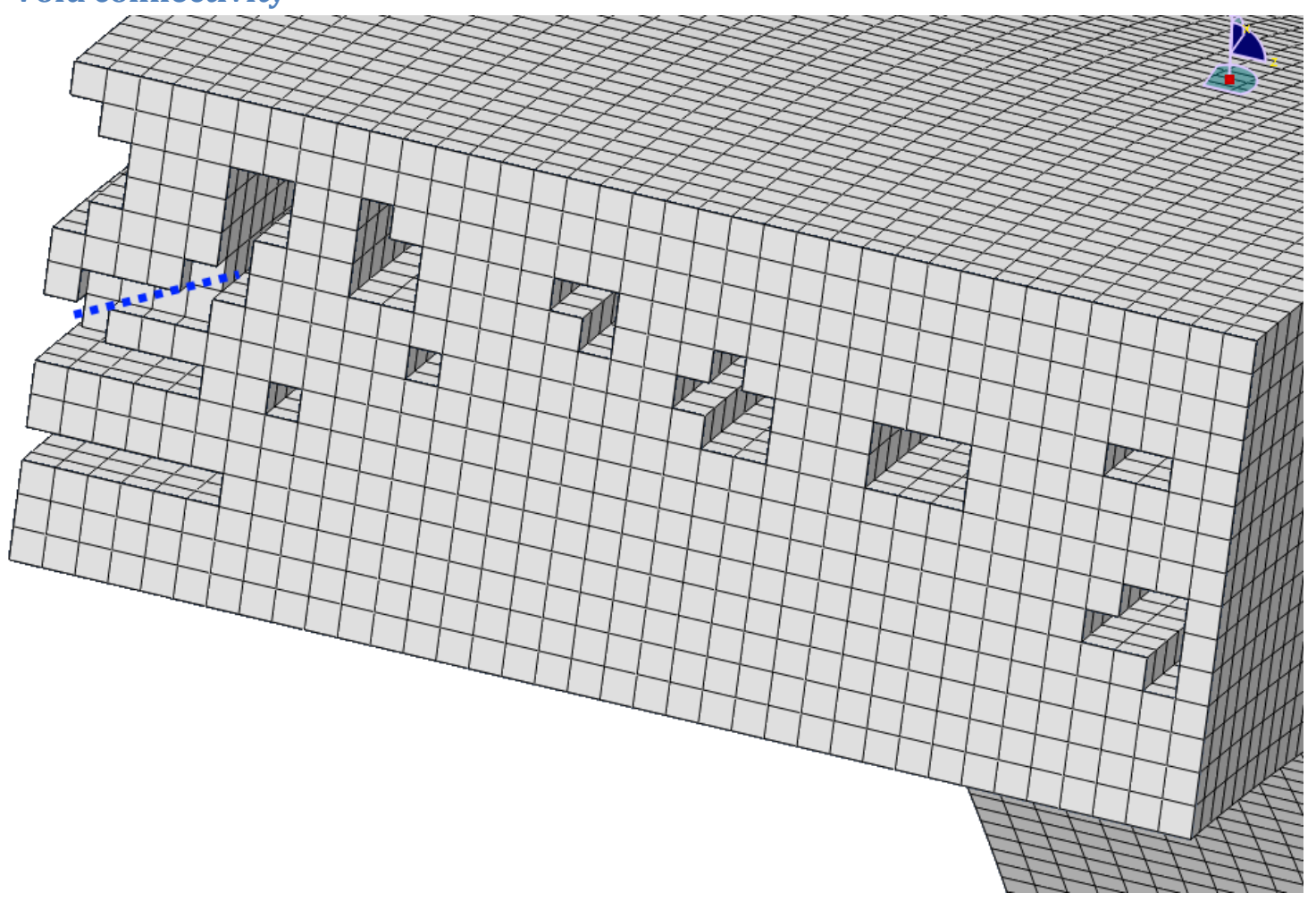

(a): Link between void and outside surface.

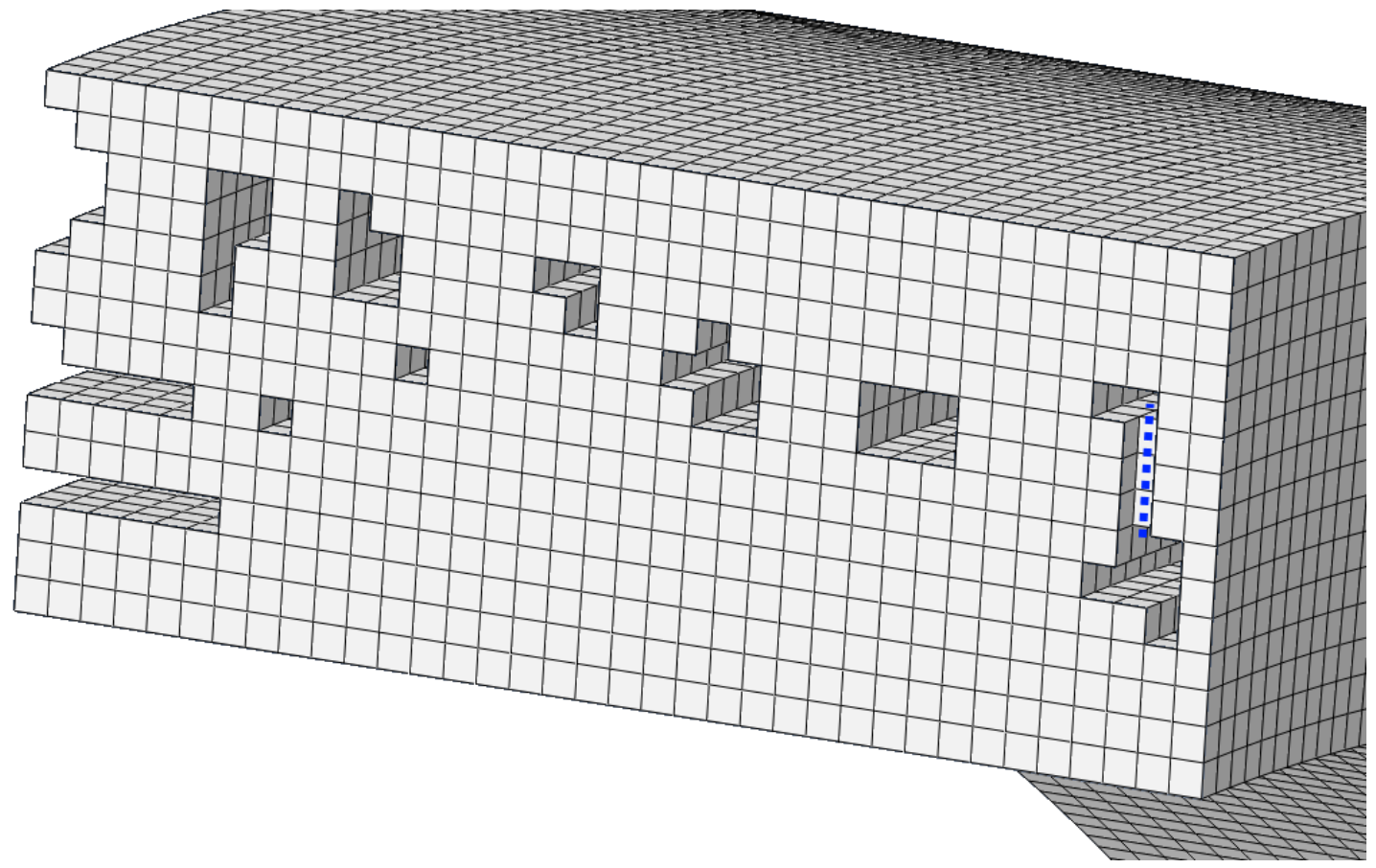

(b): Link between two internal voids at a cross section different to that shown in (a).

Fig. 11 Links created between two points within a structure using the void connectivity option during optimization of an axisymmetric structure. Only one link created for any given cross section

During the optimization process for Scheme 2, connecting links were generated between internal voids, as the example shown in Fig.(b) and for the case of two links between a void and the outer surface of the structure, as shown in Fig.(a). Further, these links were all at equal distances apart from 
each other, along the circumference of the ring, avoiding the creation of a single and much weaker cross section in generating this connectivity between voids. This is illustrated in Fig. 12.

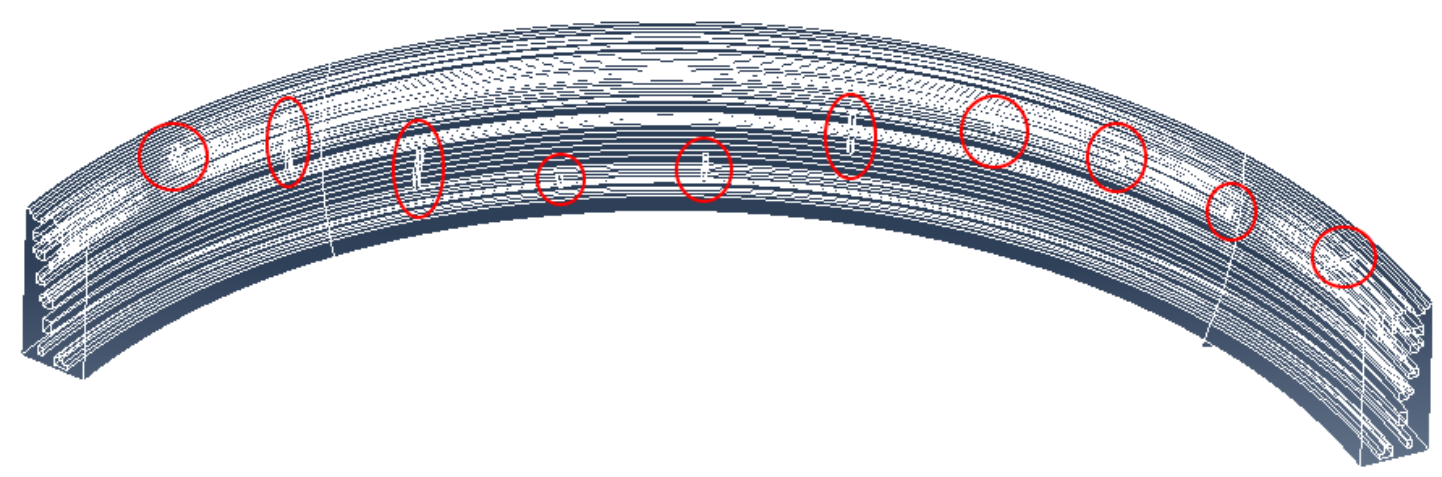

Fig. 12 Side view of the ring segment showing the connecting links (circled) generated at an equal distance along the circumferential direction

\subsubsection{Final result}

The final designs of the containment ring segments were duplicated around the circumferential direction to generate an entire ring. These designs were then converted from finite element models to Stereolithography (.stl) files. The finite element mesh produces sharp angles and edges between finite elements, especially in regions in which the finite elements had been removed by the optimization method. The solution was to apply the Laplacian smoothing algorithm (Field, 1988 and Sorkine et al., 2004) to remove the jagged edges. Final results are shown in Fig. 13. An overview of the complete geometry is shown for each of the two schemes, a clear view of the cross section before and after smoothing, and for scheme 2 an internal view of the final model, showing the voids moving through the model in the circumferential direction.

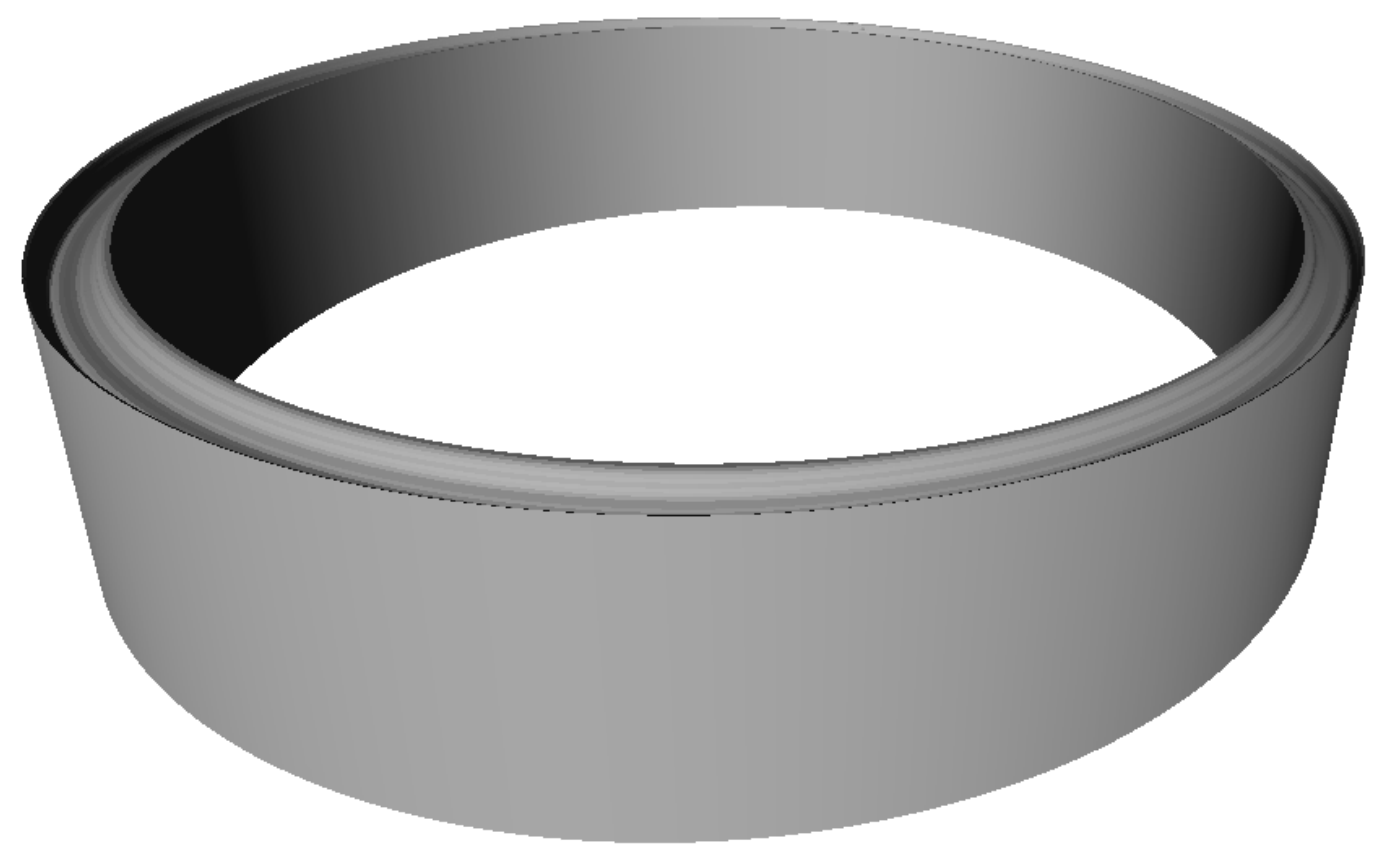

(i) Complete geometry. 


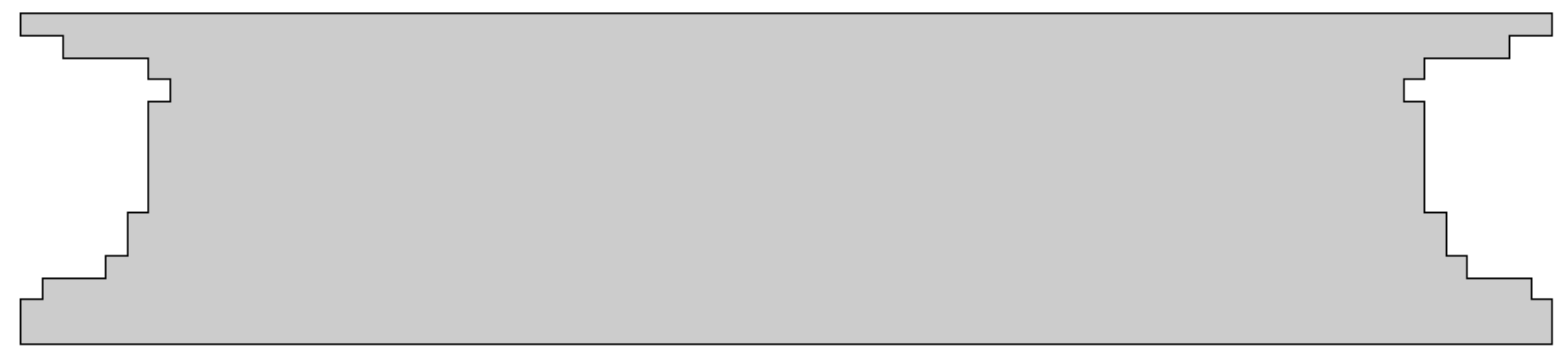

(ii) Cross-section before smoothing, from finite element model in Fig.(b).

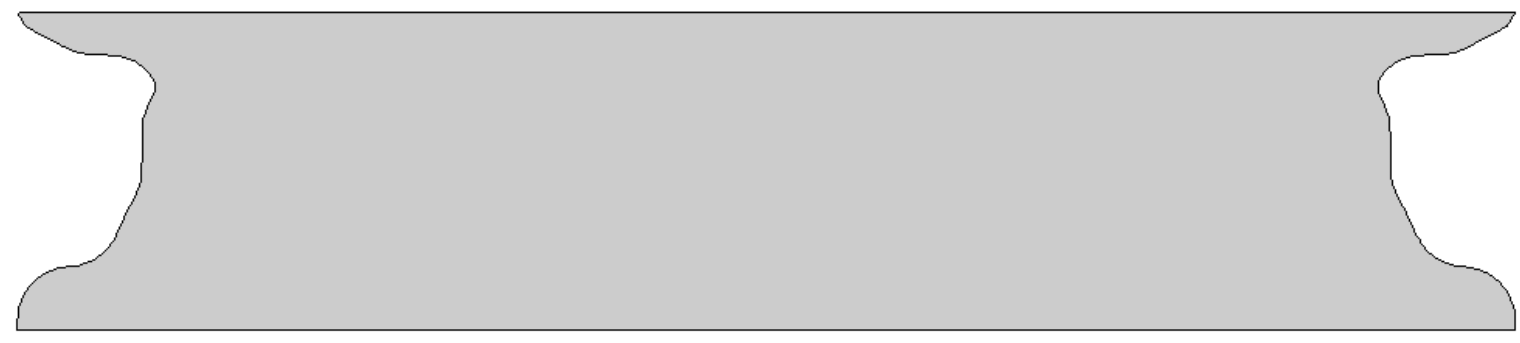

(iii) Cross-section after smoothing.

(a) Scheme 1

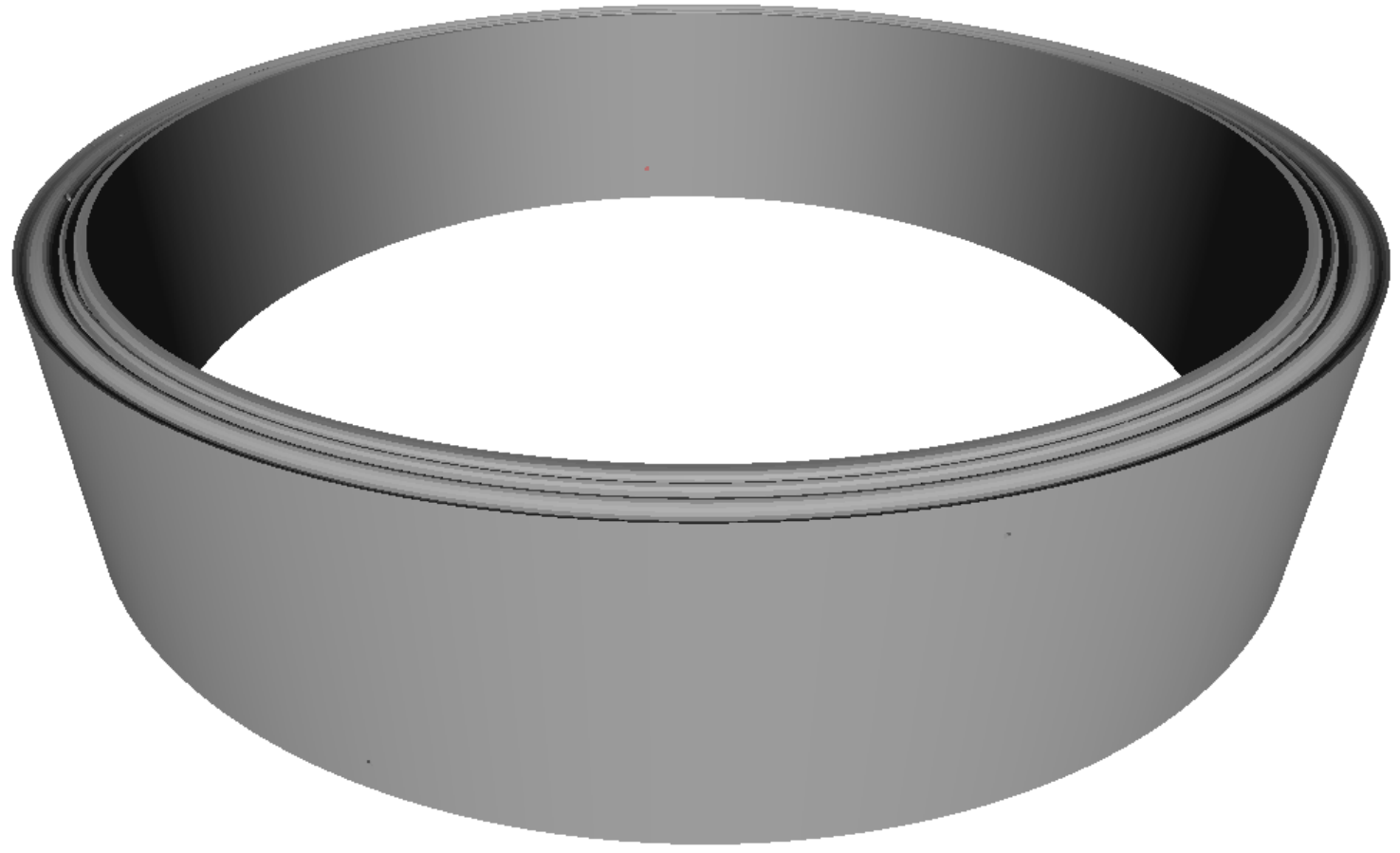

(i) Complete geometry. 


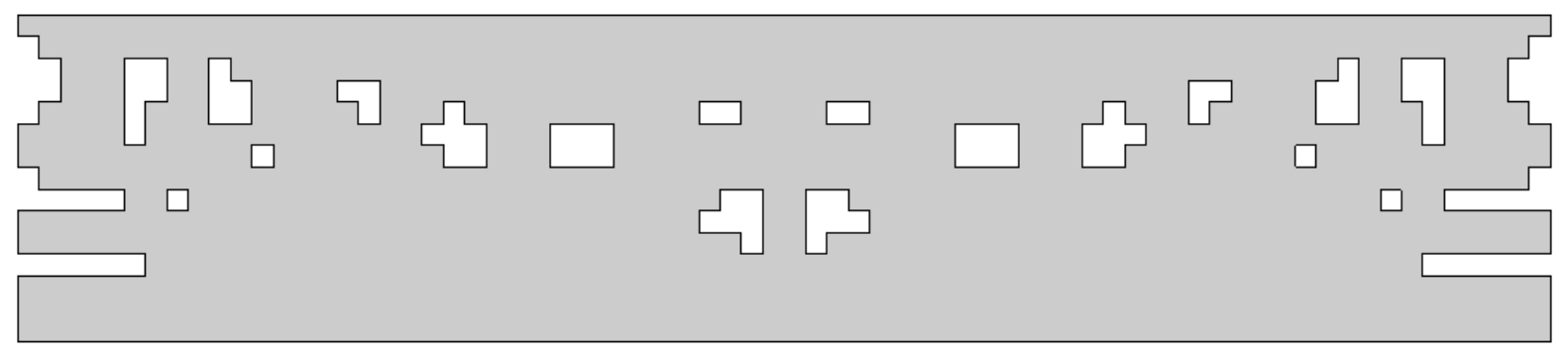

(ii) Cross-section before smoothing, from finite element model in Fig.(c).

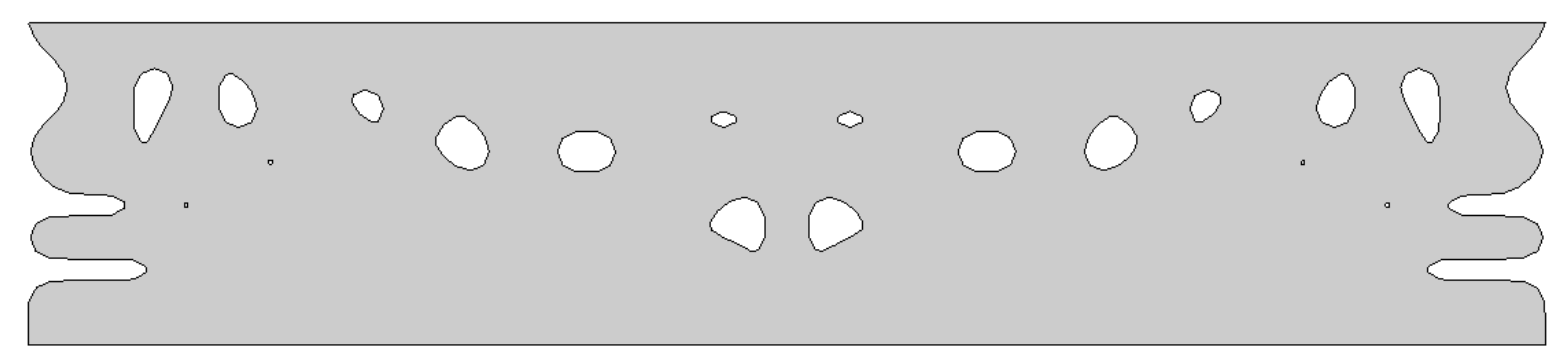

(iii) Cross-section after smoothing.

(b) Scheme 2

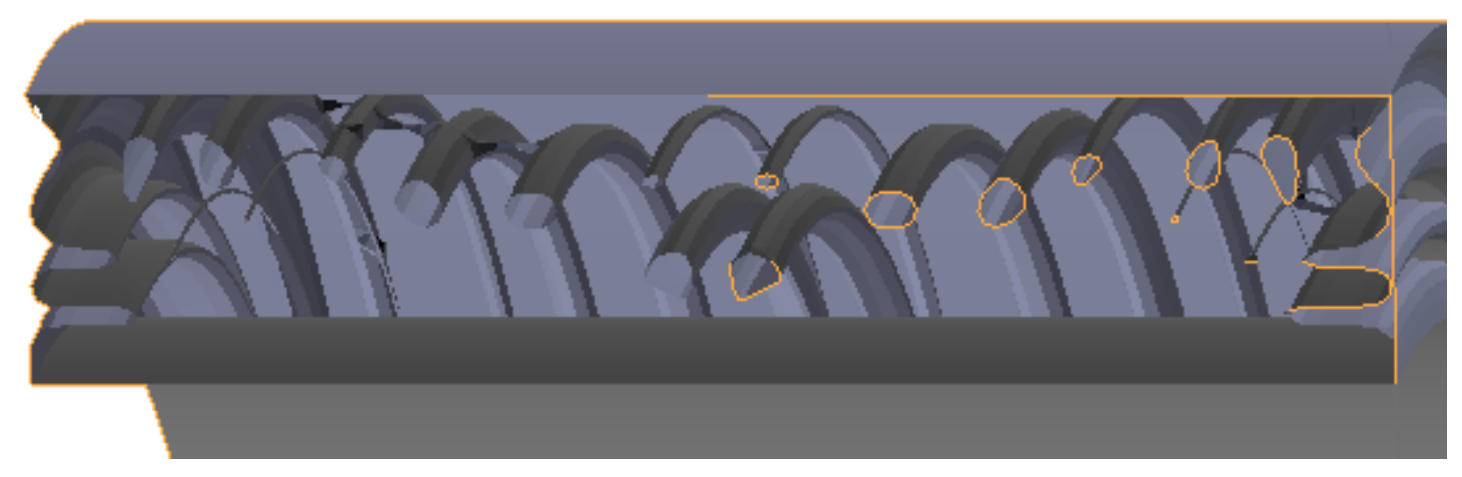

(c) Internal view of voids in the final smoothed model using optimization scheme 2.

Fig. 13 Final design geometries shown for the whole ring and from the cross section for final ring designs created using schemes 1 and 2

It is noted that if an upper void size limit is given, the total volume that can be reduced is less than if no limit is provided. This is because minimum distances between voids are also required and material is needed to keep this minimum distance. Although mechanical performance requirements also provide a natural limit to the material that can be removed, there is a geometric limit on the material that can be removed if void size limits need to be enforced. Another viewpoint on this same issue is that some elements, despite being the most efficient to remove, will be kept because they are needed to enforce void boundaries. This is shown in final results where between Fig. 13(a) and Fig. 13 (b) less material is removed from the far ends and more from the centre.

Smoothing the structure after final results had been obtained tended to change the overall volume of the structure. Although they do exist, volume preserving algorithms were not used. Further study into smoothing finite element models in preparation for manufacture is merited. In particular the 
implications for the mechanical performance of a structure given particular smoothing techniques and algorithms.

\section{Conclusions}

Three algorithms were presented which were added to the BESO optimization process to optimize an energy absorbing structure. These algorithms required basic input parameters from the designer before the optimization analysis begins. Afterward no further interaction was required and the rest of the process was automated. The results were suitable solutions to three different problems.

The first problem addressed was that of how to generate axisymmetric designs from a nonaxisymmetric finite element simulation. The designer only specified the ring's cross section, the algorithm was able to generate an optimized solution that was suitable for an impact at any location around the circumference. This was a blended solution such that if the cross section is circumferentially near or far from the impact it will be a suitable cross section to respond to the load. It was a solution optimized for a load the circumferential position of which was unknown. This algorithm can also be applied to generate an optimized prism structure with a uniform cross section.

The other two problems addressed related to voids and the implications they have for manufacturability using the SLM process. By only specifying two parameters, the designer was able to ensure that any internal voids generated by the SLM process did not exceed a maximum limit. This avoided the need to generate support structures inside a structure which would be very difficult or impossible to remove from the final build. The method was computationally simple and provided absolute control over void sizes rather than applying a penalty that might reduce sizes but could not be used to provide absolute control.

The final problem addressed was the removal of metal powder from inside a structure built using SLM. The designer was required to specify the location from which a connecting path would start and end. Once the path was generated in the design it facilitated the removal of the metal powder that would be present if the structure were built using SLM. Also shown was how this technique was integrated into a larger optimization method to allow for other procedures, such as using a structural continuity check to ensure catastrophic failure is avoided, to be used together with this one.

\section{Acknowledgement}

Funding for this research was provided by Microturbo, a member of the Safran group. Computational resources were provided by the Australian Government through the National Computational Infrastructure.

Brian G. Falzon acknowledges the financial support of Bombardier and the Royal Academy of Engineering. $X \mathrm{Wu}$ and $\mathrm{W}$ Yan acknowledge the support of Australian Research Council through the ITRH project IH130100008 and the support of the Australian Science and Industry Endowment Fund through the SIEF project RP04-153 (Aero-Engine). 


\section{References}

Allaire, G., Jouve, E. and Michailidis, G. (2016) Thickness control in structural optimization via a level set method. Structural and Multidisciplinary Optimization, 53(6):1349-1382 DOI: 10.1007/s00158016-1453-y.

Alexandersen, J. and Lazarov, B.S. (2015) Topology optimisation of manufacturable microstructural details without length scale separation using a spectral coarse basis preconditioner. Computer Methods in Applied Mechanics and Engineering, 290:156-182, DOI:10.1016/j.cma.2015.02.028.

ASM International (1967) Inconel Alloy 625. Alloy Digest.

Bendsøe, M.P. (1989) Optimal shape design as a material distribution problem. Structural optimization, 1(4):193-202, DOI:10.1007/BF01650949.

Brackett, D., Ashcroft, I., and Hague, R. (August 2001) Topology optimization for additive manufacturing. In Twenty Second Solid Freeform Fabrication (SFF) Symposium, pages 348-362, Austin, USA, University of Texas.

Brooks, W., Sutcliffe, C., Cantwell, W., Fox, P., Todd, J., and Mines, R. (August 2005) Rapid design and manufacture of ultralight cellular materials. In Sixteenth Solid Freeform Fabrication (SFF) Symposium, pages 231-241, Austin, USA, University of Texas.

Bruns, T.E. and Tortorelli, D.A. (2001) Topology optimization of non-linear elastic structures and compliant mechanisms. Computer Methods in Applied Mechanics and Engineering, 190(26-27):34433459, DOI:10.1016/S0045-7825(00)00278-4.

Bruns, T.E. and Tortorelli, D.A. (2003) An element removal and reintroduction strategy for the topology optimization of structures and compliant mechanisms. International Journal for Numerical Methods in Engineering, 57(10):1413-1430, DOI:10.1002/nme.783.

Challis, V.J., Roberts, A.P., Grotowski, J.F., Zhang, L.-C., and Sercombe, T.B. (2010) Prototypes for Bone Implant Scaffolds Designed via Topology Optimization and Manufactured by Solid Freeform Fabrication. Advanced Engineering Materials, 12(11):1106-1110, DOI:10.1002/adem.201000154.

Chen, S., Wang, M.Y., and Liu, A.Q. (2008) Shape feature control in structural topology optimization. Computer-Aided Design, 40(9):951-962, DOI:10.1016/j.cad.2008.07.004.

Chen, Y. (2006) A Mesh-Based Geometric Modeling Method for General Structures. ASME Conference Proceedings, 2006(42578):269-281, DOI:10.1115/DETC2006-99513.

Chu, C., Graf, G., and Rosen, D.W. (2008) Design for Additive Manufacturing of Cellular Structures. Computer-Aided Design and Applications, 5(5):686-696, DOI:10.3722/cadaps.2008.686-696.

Chu, D.N., Xie, Y.M., Hira, A., and Steven, G.P. (1996) Evolutionary structural optimization for problems with stiffness constraints. Finite Elements in Analysis and Design, 21(4):239-251, DOI:10.1016/0168874X(95)00043-S. 
Deaton, J. and Grandhi, R.V. (2013) A survey of structural and multidisciplinary continuum topology optimization: post 2000. Structural and Multidisciplinary Optimization, 49(1):1-38, DOI:10.1007/s00158-013-0956-z.

Du, J. and Olhoff, N. (2007) Topological design of freely vibrating continuum structures for maximum values of simple and multiple eigenfrequencies and frequency gaps. Structural and Multidisciplinary Optimization, 34(2):91-110, DOI:10.1007/s00158-007-0101-y.

Field, D.A. (1988) Laplacian smoothing and Delaunay triangulations, Communications in Applied Numerical Methods, 4:709-712.

Forsberg, J. and Nilsson, L. (2007) Topology optimization in crashworthiness design. Structural and Multidisciplinary Optimization, 33(1):1-12, DOI:10.1007/s00158-006-0040-z.

Guest, J.K. (2009) Imposing maximum length scale in topology optimization. Structural and Multidisciplinary Optimization, 37(5):463-473, DOI:10.1007/s00158-008-0250-7.

Guest, J.K., Prévost, J.H., and Belytschko, T. (2004) Achieving minimum length scale in topology optimization using nodal design variables and projection functions. International Journal for Numerical Methods in Engineering, 61(2):238-254, DOI:10.1002/nme.1064.

Haber, R.B., Jog, C.S., and Bendsøe, M.P. (1996) A new approach to variable-topology shape design using a constraint on perimeter. Structural optimization, 11(1):1-12, DOI:10.1007/BF01279647.

Hagg, A.C. and Sankey, G.O. (1974) The Containment of Disk Burst Fragments by Cylindrical Shells. Journal of Engineering for Power, 96(2):114-123, DOI:10.1115/1.3445758.

Haynes International Inc. (2001) Haynes 625 alloy. Technical Report H-3073D, Kokomo, Indiana 469049013 (USA). <http://www.haynesintl.com/pdf/h3073.pdf> Retrieved 28-Dec-2012.

Hopkinson, N., Hague, R.J.M., and Dickens, P.M. (editors) (2006) Rapid Manufacturing: An Industrial Revolution for the Digital Age. John Wiley \& Sons, Ltd, West Sussex PO19 8SQ, England, ISBN:978-04700-3399-9, DOI:10.1002/0470033991.

Huang, X. and Xie, Y.M. (2007) Convergent and mesh-independent solutions for the bi-directional evolutionary structural optimization method. Finite Elements in Analysis and Design, 43(14):10391049, DOI:10.1016/j.finel.2007.06.006.

Huang, X. and Xie, Y.M. (2010) A further review of ESO type methods for topology optimization. Structural and Multidisciplinary Optimization, 41(5):671-683, DOI:10.1007/s00158-010-0487-9.

Huang, X., Xie, Y.M., and Burry, M.C. (2006) A New Algorithm for Bi-Directional Evolutionary Structural Optimization. JSME international journal. Series C, 49(4):1091-1099, DOI:10.1299/jsmec.49.1091.

Huang, X., Xie, Y.M., and Lu, G. (2007) Topology optimization of energy-absorbing structures. International Journal of Crashworthiness, 12(6):663-675, DOI:10.1080/13588260701497862.

Jarvis, T. (2013). Private communication. 
Jung, D. and Gea, H.C. (2006) Design of an energy-absorbing structure using topology optimization with a multimaterial model. Structural and Multidisciplinary Optimization, 32(3):251-257, DOI:10.1007/s00158-006-0011-4.

Kato, J., Hoshiba, H., Takase, S., Terada, K., Kyoya, T. (2015) Analytical sensitivity in topology optimization for elastoplastic composites, Structural and Multidisciplinary Optimization, 52(3):507526.

Kobayashi, T., Simons, J.W., Brown, C.S., and Shockey, D.A. (2008) Plastic flow behavior of Inconel 718 under dynamic shear loads. International Journal of Impact Engineering, 35(5):389-396, DOI:10.1016/j.jimpeng.2007.03.005.

Li, Q., Steven, G.P., Xie, Y.M., and Querin, O.M. (2004) Evolutionary topology optimization for temperature reduction of heat conducting fields. International Journal of Heat and Mass Transfer, 47(23):5071-5083, DOI:10.1016/j.ijheatmasstransfer.2004.06.010.

Lin, S. and Kernighan, B.W. (1973) An Effective Heuristic Algorithm for the Traveling-Salesman Problem. Operations Research, 21(2):498-516.

Maute, K., Schwarz, S., and Ramm, E. (1998) Adaptive topology optimization of elastoplastic structures. Structural optimization, 15(2):81-91, DOI:10.1007/BF01278493.

Mayer, R.R., Kikuchi, N., and Scott, R.A. (1996) Application of topological optimization techniques to structural crashworthiness. International Journal for Numerical Methods in Engineering, 39(8):13831403, DOI:10.1002/(SICI)1097-0207(19960430)39:8<1383::AID-NME909>3.0.CO;2-3.

Naing, M.W., Chua, C.K., Leong, K.F., and Wang, Y. (2005) Fabrication of customised scaffolds using computer-aided design and rapid prototyping techniques. Rapid Prototyping Journal, 11(4):249-259, DOI:10.1108/13552540510612938.

Neves, M.M., Rodrigues, H., and Guedes, J.M. (1995) Generalized topology design of structures with a buckling load criterion. Structural optimization, 10(2):71-78, DOI:10.1007/BF01743533.

Pedersen, C.B.W. (2003) Topology optimization design of crushed 2D-frames for desired energy absorption history. Structural and Multidisciplinary Optimization, 25(5-6):368-382, DOI:10.1007/s00158-003-0282-y.

Pereira, J.M. and Lerch, B.A. (2001) Effects of heat treatment on the ballistic impact properties of INCONEL718 for jet engine fan containment applications. International Journal of Impact Engineering, 25(8):715-733, DOI:10.1016/S0734-743X(01)00018-5.

Poulsen, T.A. (2003) A new scheme for imposing a minimum length scale in topology optimization. International Journal for Numerical Methods in Engineering, 57(6):741-760, DOI:10.1002/nme.694.

Prasad, B. and Emerson, J.F. (1984) Optimal structural remodeling of multi-objective systems. Computers \& Structures, 18(4):619-628, DOI:10.1016/0045-7949(84)90007-5. 
Querin, O.M., Young, V., Steven, G.P., and Xie, Y.M. (2000) Computational efficiency and validation of bi-directional evolutionary structural optimisation. Computer Methods in Applied Mechanics and Engineering, 189(2):559-573, DOI:10.1016/S0045-7825(99)00309-6.

Rosen, D.W. (2007) Computer-aided design for additive manufacturing of cellular structures. Computer-Aided Design and Applications, 4(1-6):585-594, DOI:10.1080/16864360.2007.10738493.

Rozvany, G.I.N., Zhou, M., and Birker, T. (1992) Generalized shape optimization without homogenization. Structural optimization, 4(3-4):250-252, DOI:10.1007/BF01742754.

Rozvany, G.I.N. (2009) A critical review of established methods of structural topology optimization. Structural and Multidisciplinary Optimization, 37(3):217-237. DOI:10.1007/s00158-007-0217-0.

Samanta, A., Teli, M., and Singh, R.K. (March 2012) Surface integrity in laser assisted mechanical micromachining of (LAMM) of Inconel 625. In Proceedings of International Conference on Micromanufacturing, Chicago, USA.

Schroeder, C., Regli, W.C., Shokoufandeh, A., and Sun, W. (2005) Computer-aided design of porous artifacts. Computer-Aided Design, 37(3):339-353, DOI:10.1016/j.cad.2004.03.008.

Sorkine, O., Cohen-Or, D., Lipman, Y., Alexa, M., Rössl, C., and Seidel, H.-P. (2004) Laplacian surface editing. In Proceedings of the 2004 Eurographics/ACM SIGGRAPH Symposium on Geometry Processing, SGP'04, pages 175-184, New York, NY, USA, ACM.

Special Metals Corporation (Jan 2006) Inconel alloy 625. Technical Report SMC-063, Huntington, WV 25705-1771 (USA). <http://www.specialmetals.com/documents/Inconel\%20alloy\%20625.pdf> Retrieved 30-Dec-2012.

Stojanov, D., Falzon, B.G., Wu, X., and Yan, W. (2016) Implementing a structural continuity constraint and a halting method for the topology optimization of energy absorbers. Structural and Multidisciplinary Optimization, 1-20, DOI:10.1007/s00158-016-1451-0.

Sun, W., Starly, B., Nam, J., and Darling, A. (2005) Bio-CAD modeling and its applications in computeraided tissue engineering. Computer-Aided Design, 37(11):1097-1114, DOI:10.1016/j.cad.2005.02.002.

Wallin, M., Jönsson, V., Wingren, E. (2016) Topology optimization based on finite strain plasticity. Structural and Multidisciplinary Optimization, 54(4):783-793.

Wang, H., Chen, Y., and Rosen, D.W. (2005) A Hybrid Geometric Modeling Method for Large Scale Conformal Cellular Structures. ASME Conference Proceedings, 2005(47403):421-427, DOI:10.1115/DETC2005-85366.

Watts, D.M. and Hague, R.J. (August 2006) Exploiting the design freedom of rm. In Seventeenth Solid Freeform Fabrication (SFF) Symposium, pages 656-667, Austin, USA, University of Texas.

Wu, C.-Y. and Tseng, K.-Y. (2010) Topology optimization of structures using modified binary differential evolution. Structural and Multidisciplinary Optimization, 42(6):939-953, DOI:10.1007/s00158-0100523-9. 
Yang, X.Y., Xie, Y.M., Liu, J.S., Parks, G.T., and Clarkson, P.J. (2002) Perimeter control in the bidirectional evolutionary optimization method. Structural and Multidisciplinary Optimization, 24(6):430-440, DOI:10.1007/s00158-002-0256-5.

Yoon, G.H. and Kim, Y.Y. (2007) Topology optimization of material-nonlinear continuum structures by the element connectivity parameterization. International Journal for Numerical Methods in Engineering, 69(10):2196-2218, DOI:10.1002/nme.1843.

Yuge, K. and Kikuchi, N. (1995) Optimization of a frame structure subjected to a plastic deformation. Structural optimization, 10(3):197-208, DOI:10.1007/BF01742592.

Zhang, G., Li, L., Khandelwal, K. (2016) Topology optimization of structures with anisotropic plastic materials using enhanced assumed strain elements, Structural and Multidisciplinary Optimization. Published online on 12 December 2016. 